\title{
Contour length fluctuations and constraint release in entangled polymers: Slip-spring simulations and their implications for binary blend rheology
}

Daniel J. Read, Maksim E. Shivokhin, and Alexei E. Likhtman

Citation: Journal of Rheology 62, 1017 (2018); doi: 10.1122/1.5031072

View online: https://doi.org/10.1122/1.5031072

View Table of Contents: http://sor.scitation.org/toc/jor/62/4

Published by the The Society of Rheology

\section{Articles you may be interested in}

Shear thinning in dilute and semidilute solutions of polystyrene and DNA

Journal of Rheology 62, 845 (2018); 10.1122/1.5010203

The transient behavior of soft glassy materials far from equilibrium

Journal of Rheology 62, 869 (2018); 10.1122/1.5024701

A new rate-independent tensorial model for suspensions of noncolloidal rigid particles in Newtonian fluids Journal of Rheology 62, 889 (2018); 10.1122/1.4995817

Molecular simulations of asphalt rheology: Application of time-temperature superposition principle Journal of Rheology 62, 941 (2018); 10.1122/1.4996919

Role of inertia and thixotropy in start-up flows of aging soft materials: Transient dynamics and shear banding in a rate-controlled flow field

Journal of Rheology 62, 1001 (2018); 10.1122/1.5023305

Time-temperature equivalence in a PVA dual cross-link self-healing hydrogel

Journal of Rheology 62, 991 (2018); 10.1122/1.5029466

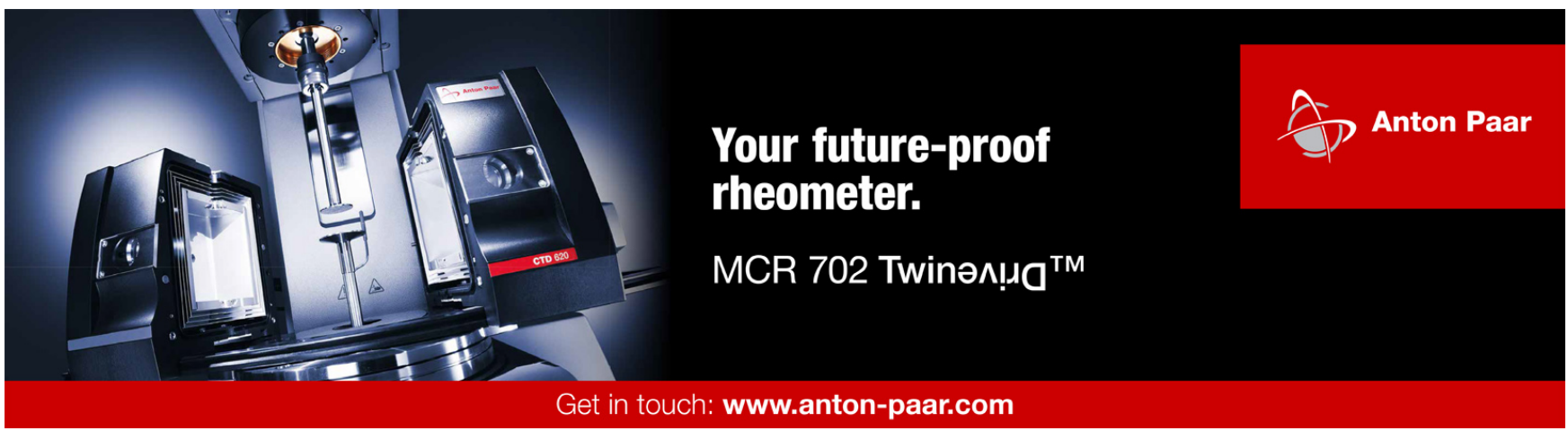




\title{
Contour length fluctuations and constraint release in entangled polymers: Slip-spring simulations and their implications for binary blend rheology
}

\author{
Daniel J. Read, ${ }^{1, a)}$ Maksim E. Shivokhin, ${ }^{2}$ and Alexei E. Likhtman ${ }^{3}$ \\ ${ }^{1}$ School of Mathematics, University of Leeds, Leeds LS2 9JT, United Kingdom \\ ${ }^{2}$ Advanced Characterisation Department, ExxonMobil Chemical Company, 5200 Bayway Drive, \\ Baytown, Texas 77520 \\ ${ }^{3}$ School of Mathematical and Physical Sciences, University of Reading, Reading RG6 6AX, United Kingdom
}

(Received 28 March 2018; final revision received 12 June 2018; published 13 July 2018)

\begin{abstract}
We study the interaction between constraint release and contour length fluctuations in well entangled polymers, by means of a set of slipspring simulations in which the rate of constraint release is precisely controlled. In the present simulations, a fraction $f=0.9$ of the slipsprings undergo constraint release, while the remaining slip-springs do not, as an idealized model to mimic the constraint release environment of a bidisperse polymer melt. Both populations of slip-springs allow reptation of the chain. Our analysis of the data indicates the time and parameter regions in which contour length fluctuations occur effectively along a thin tube, or along a diluted fat tube. We predict the parameters for which case each is observed and the rate of relaxation due to contour length fluctuations in each region. Finally, we draw the implications of the simulations for bidisperse blend rheology, by revisiting the classic "Viovy diagram" for such melts. We relocate some of the lines on the original diagram, and identify new regimes, based on the physics and quantitative information supplied by the simulation data. In particular, we identify a new region in the diagram in which along-tube motion of the long chains is predominantly along the contour of the thin tube, yet contour length fluctuations occur in the fat tube, resulting in an acceleration of the terminal relaxation. We successfully and quantitatively locate a wide range of literature data on our redrawn diagram. (C 2018 Author(s). All article content, except where otherwise noted, is licensed under a Creative Commons Attribution (CC BY) license (http://creativecommons.org/licenses/by/4.0/). https://doi.org/10.1122/1.5031072
\end{abstract}

\section{INTRODUCTION}

The dynamics of entangled polymers have intrigued soft matter scientists for at least half a century. Entanglements between the long molecules severely impede the Brownian dynamics of the molecules, giving extremely long times for molecular relaxation, manifest in the viscoelastic nature of polymeric liquids. The dominant theoretical picture remains the "tube model" of de Gennes [1] and Doi and Edwards [2]: Entanglements restrict the molecules to a tubelike region of space surrounding the polymer (though alternative pictures such as "slip-link" models [3-7] provide useful computational schemes). Whichever model is used, the primary chain motion for monodisperse linear polymers is considered to be "reptation"-chain diffusion along a contour defined by the entanglements (the "tube contour," in the tube model). Quantitative description of these dynamics is possible, but requires the inclusion of two additional processes to the theory: "Contour length fluctuation" (CLF) occurs because flexible polymers have the freedom to vary their projected length along the tube contour, allowing chain ends to rapidly relax; "Constraint release" (CR) occurs because the surrounding molecules

\footnotetext{
a) Author to whom correspondence should be addressed; electronic mail: d.j.read@leeds.ac.uk

(c) Author(s) 2018.C. (C)

J. Rheol. 62(4), 1017-1036 July/August (2018)
}

are also in constant random motion, and so are able to release and renew their entanglement constraints [8]. Likhtman and McLeish [9] presented a very successful theory describing the linear rheology of monodisperse linear polymers, which combines all of the above modes of motion. For such monodisperse polymers, CR provides only a relatively small perturbation because CR is slow, occurring only on a timescale of terminal chain relaxation (CR relaxes stress, and so gives roughly a factor of two change in the dominant stress relaxation time, but virtually no change in diffusion rate or end-toend relaxation time).

Given the above (extremely complete) picture, it is perhaps surprising that the second simplest polymeric liquid, a binary mixture of polymer chains of two lengths, remains a topic for active research. Complications arise because CR from the shorter chains is fast, and no longer a small perturbation for the slower, longer chain dynamics. This is of crucial and practical importance, since all industrial plastic materials are polydisperse, containing a broad mixture of polymer lengths, giving a spectrum of CR times. For predicting linear viscoelasticity of these materials, researchers have used variants on the "double reptation" scheme [10], but this usually makes the bold assumption that the viscoelastic response can be calculated from the relaxation of the separate components considered on their own. This implies that the terminal relaxation time of long chains is entirely unaffected by the shorter ones. Although this seems to work for 


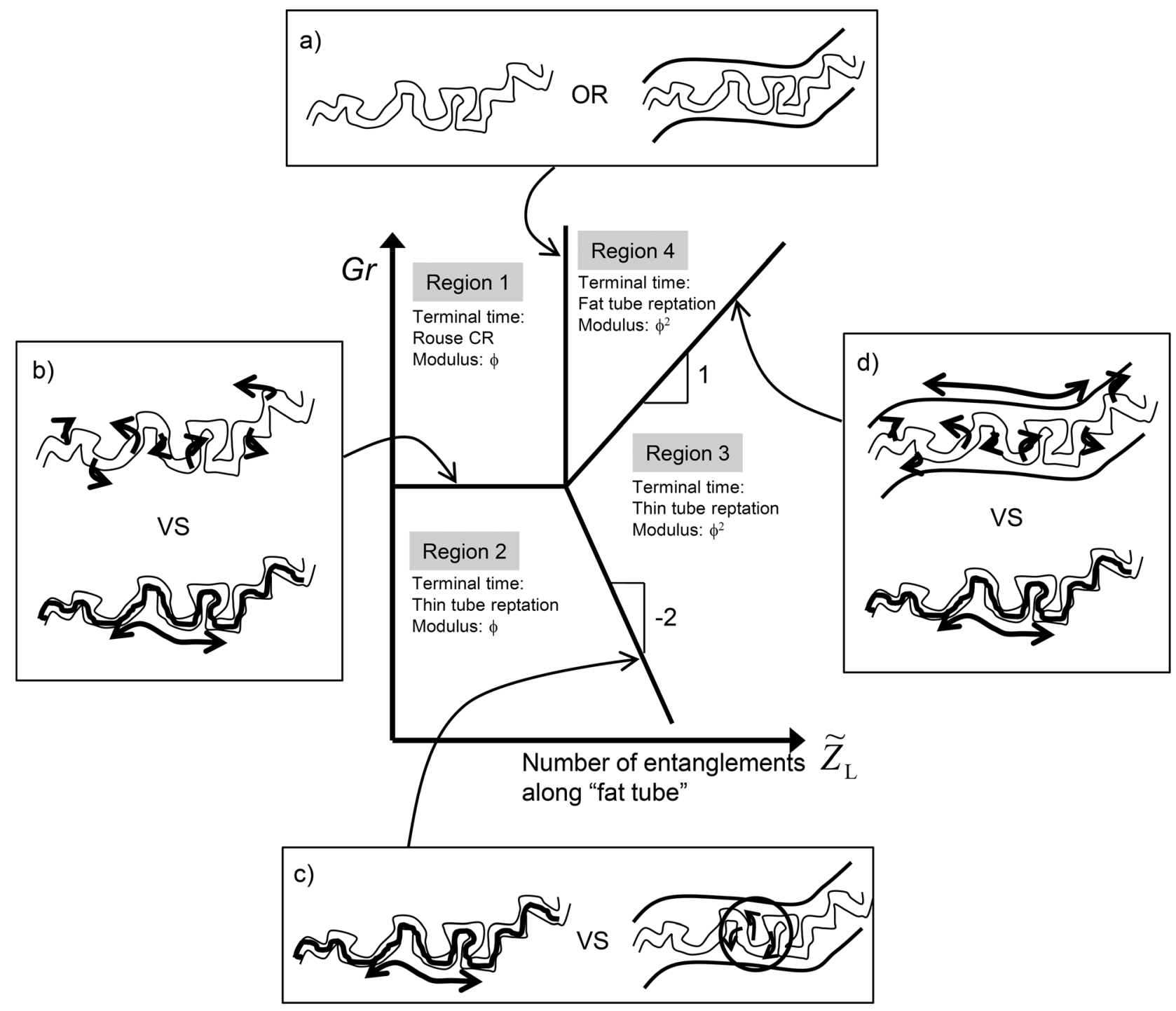

FIG. 1. Schematic Viovy diagram [18] indicating, in each region, the process governing terminal relaxation and how the terminal modulus scales with fraction $\phi$ of long chains. In region 1, dilute long chains relax terminally through CR; in region 2 they relax by reptation. In regions 3 and 4 , long chains are selfentangled; hence the terminal modulus scales as $\phi^{2}$; in region 3, reptation along the thin tube dominates, while in region 4 motion along the fat tube, occurring due to $\mathrm{CR}$ of the thin tube, is predicted to accelerate the terminal reptation of the long chains. Also shown are cartoons depicting the processes that compete at the boundaries between regions. Line (a): at $\tilde{Z}=1$ is the boundary between long chains entangled with only short chains (so only a thin tube is needed), or long chains also entangled with other long chains (giving the fat tube). Line (b): at $G r \approx 1$, CR Rouse relaxation (top) competes with reptation along the thin tube (bottom). Line (c): Reptation along the thin tube (left) competes with local CR-induced equilibration of thin tube in one fat tube segment (right). Line (d): Reptation along the thin tube (bottom) competes with reptation along the fat tube driven by CR (top).

some materials, it is demonstrably false for many binary polymer mixtures, see, e.g., $[8,11-17]$. This suggests a critical question to be addressed in this paper by means of sliplink simulations: Does faster CR from short chains affect the motion via reptation or CLF of longer chains, and how does this occur?

One way to think of the long chains in a binary mixture is to consider them as being trapped within two tubes: A thin tube representing entanglements with all chains, and a fat tube representing entanglements with long chains only (see the cartoons in Fig. 1). Fast CR from the short chains allows the thin tube to reconfigure and relax. Considering only reptation and CR (but ignoring CLF) Viovy et al. [18] presented an appealing diagram (Fig. 1) illustrating the rich set of dynamical regimes for the long chains. The horizontal axis is the number of entanglements along the fat tube- to the left of the diagram the long chains are too short, or too dilute, to entangle with other long chains. The vertical axis is the "Graessley" parameter, $G r=Z_{L} / Z_{S}^{3}$, where $Z_{L}$ and $Z_{S}$ are the number of entanglements along the long and short chains, respectively $[19,20]$. $G r$ measures the relative rates of relaxation by CR or by reptation. In region 1 of the Viovy diagram, dilute long chains relax terminally through $\mathrm{CR}$; in region 2 they relax by reptation. In regions 3 and 4, long chains are self-entangled; in region 3, reptation along the thin tube dominates, while in region 4 motion along the fat tube, occurring due to $\mathrm{CR}$ of the thin tube, is predicted to accelerate the terminal reptation of the long chains. In region 3 , the relaxation time of the long chains is therefore predicted to be completely independent of the short chain concentration or length; but experiments contradict this, and dilution with short chains does speed up (e.g., by a factor of 
5) the long chain relaxation across blends found in region 3 of the diagram. Specific examples of such blends, clearly in region 3, which exhibit this speeding up include mixtures of $480 \mathrm{k}$ and $34 \mathrm{k}$ Polyisoprene [11], mixtures of 550000 and 20000 polybutadiene [14,15], and mixtures of 294000 and 83000 or 161000 polystyrene [16].

In considering this problem, Park and Larson [14,15] presented a theory in which they considered reptation to occur either along the thin tube (at low values of the Graessley parameter) or along the fat tube (at high values of the Graessley parameter), with the critical value of the Graessley parameter found to be in the region of 0.1 . This work contradicts the physical picture proposed by Viovy et al. because Park and Larson assume that motion along the fat tube proceeds at a rate determined by monomeric friction only, rather than at a rate dictated by $\mathrm{CR}$ events. They also do not have a means to transition smoothly between the two different reptation regimes. Nevertheless, the work of Park and Larson $[14,15]$ is useful because it highlights that there is a speeding up of long chains upon dilution with shorter chains, and that it is impossible to account for this when considering only reptation along the thin tube.

A possible solution to the problem, proposed recently $[11,12]$, is to include the effects of CLFs. In particular, we note that CLF modes in the fat tube are possible even in region 3 of the Viovy diagram, and that these provide a candidate mechanism for short chains to speed up the relaxation of long chains. Slip-link simulations [3] present an ideal platform to test some of these ideas and can be mapped onto the tube model as demonstrated in our earlier paper [21]. Here we focus specifically on the effect of CR on the CLF process, using a set of simulations designed for this purpose.

We present first the simulations, their results, and our analysis of them in terms of tube theory. We then seek to draw out the implications of the simulations for experimental studies of binary blends. In particular, we will re-examine the Viovy diagram in the light of CLFs of both short and long chains. This will result in a redrawn Viovy diagram, in which existing lines are repositioned based on the numerical results from simulations, and in which new lines are added to delineate regions with different CLF behavior. We will demonstrate that literature data for binary blends can be successfully mapped on to our redrawn diagram.

Throughout the paper, we shall assume a dilution exponent of $\alpha=1$. This is the natural assumption in slip-link models, where entanglements are considered as binary events. It also appears to be a reasonable assumption for real polymeric liquids (see, e.g., [22]).

We briefly note, here, that it is not a priori obvious that it is possible to perform the mapping between the slip-spring model and the tube model, alluded to above and used in our earlier paper [21]. Certainly, chain motions in a fixed tube (or fixed array of slip-spring constraints), i.e., reptation and CLF, should be similar in both models. However, it is not clear that all the chain motions induced by $\mathrm{CR}$, as envisaged in the physics of fat and thin tubes, should have a direct analog in the slip-spring model. To some extent, the proof of the mapping is in its success in the previous work [21] and the results

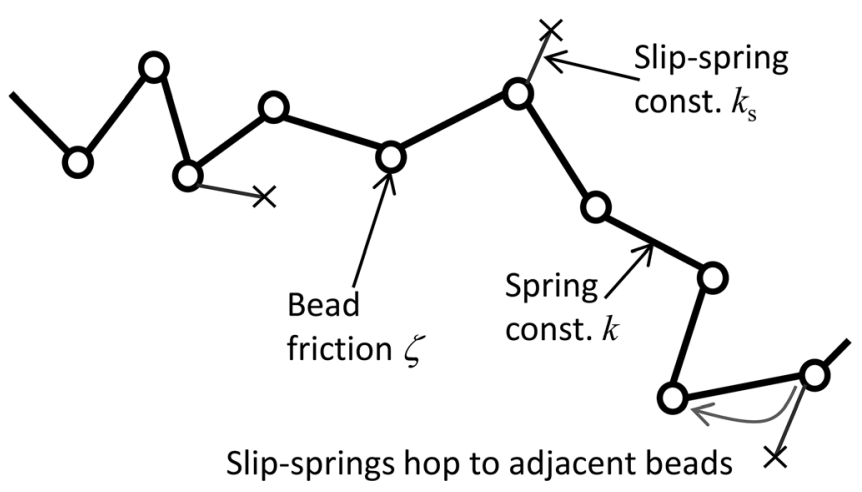

FIG. 2. Schematic diagram of the slip-spring model employed in this work. The simulations use Rouse chains comprising frictional beads (with stochastic thermal noise) connected by springs. Entanglements are modeled using slip-springs, which attach beads to anchoring points, and which hop stochastically between beads.

described below. We shall return to a brief discussion of why this mapping might be successful in the concluding section.

\section{SIMULATIONS}

Our simulations are based on the "slip-spring" model of Likhtman [3], illustrated in Fig. 2. The simulations comprise Rouse chains with $N=512$ frictional beads (with associated fluctuating thermal force) connected by $N-1$ springs. Entanglements are modeled by slip-links randomly distributed along the chain. Every slip-link is connected by a virtual spring to an anchoring point, and so are given the name slipsprings. The positions of the anchoring points are determined to preserve, on average, the unperturbed Gaussian statistics of the chain conformations at all length scales. Slip-springs move between neighboring beads through Metropolis Monte Carlo moves (such that two slip-springs are not permitted to attach to the same bead): This allows both reptation and CLF to occur. Setting the friction constant per bead $\zeta=1$, the polymeric spring step length $b=1$ and the thermal energy $k_{\mathrm{B}} T=1$ give a simulation time unit $\zeta b^{2} / k_{\mathrm{B}} T=1$, which is proportional to the local bead relaxation time. We use a time step $d t=0.05$. The spring constant between beads is then $k=3 k_{\mathrm{B}} T / b^{2}=3$. We employ the "standard" Likhtman parameters of $N_{e, s s}=4$ chain beads per slip-spring (on average) and slip-spring constant $k_{s}=3 k_{\mathrm{B}} T / N_{s} b^{2}$ with $N_{s}=0.5$ ( $N_{s}$ is the effective "number of beads" in a slip-spring). These values of $N_{e, s s}$ and $N_{s}$ were used in the original Likhtman slip-spring paper [3] (where they were shown to give good representation of rheology, neutron spin echo, and diffusion data) and have since been used in many publications using the slip-spring model (see, e.g., [21,23,24]).

Since the slip-springs are not pointlike, but rather allow the chain to fluctuate about the slip-spring anchor point, this smooths out the contour of the equivalent tube model (so that the tube contour is smoother than the set of vectors between slip-links). This means that the effective number of beads per entanglement in the equivalent tube model is $N_{e}>N_{e, s s}$, which we previously estimated as $N_{e}=4.89$ for the standard Likhtman parameters above [21] (as detailed in 
Appendix A), giving the number of tube model entanglements per chain $Z \approx 105$.

We control the CR process in our simulations by random insertion and deletion of slip-springs with specified average lifetime $\tau_{\mathrm{CR}}$ (which we change from one simulation to the next). To approximate a binary blend, a fraction $f=0.1$ of the slip-springs are not deleted unless passed by the chain end; there is thus no CR for this fraction of slip-springs, which approximates the "slow" CR expected for entanglements between long chains. The remaining $90 \%$ of slipsprings are randomly inserted and deleted, representing (fast) CR from the short chains. In order to preserve the fractions of these two populations of slip-springs, if a slip-spring is deleted when passed by a chain end, another slip-spring of the same type is immediately added to a randomly selected chain end. The value of $f$ was chosen to be large enough so as to retain a reasonable number of slow slip-springs per chain (that do not undergo CR), but also to be small enough to resolve some of the intermediate CR Rouse region between thin and fat tubes.

When the CR is fast, the rapidly moving $90 \%$ of slipsprings effectively dilute the entanglement constraints, giving a dilution of the equivalent tube. As noted above, slip-springs are not pointlike objects, but allow fluctuations around their anchor point, and this in general gives $N_{e}>N_{e, s s}$. However, if $N_{e, s s}$ is increased (i.e., the slipsprings are diluted), while the slip spring strength $N_{s}=0.5$ is held constant, then the ratio $N_{e} / N_{e, s s}$ tends toward 1 . This is because the ratio of slip-spring fluctuation length to diluted tube diameter decreases as the dilution increases, i.e., the slip-springs become effectively more "pointlike" with respect to the tube diameter with increasing dilution. For this reason, a dilution to $f=0.1$ of slip-springs does not exactly correspond to the same dilution of entanglements in the equivalent tube model. This point was considered in [21], and based on these considerations (as detailed in Appendix A), we find that a dilution $f=0.1$ corresponds to a dilution $\phi_{f}=0.12$ of entanglements in the equivalent tube model [21]; hence, the number of entanglements along the "fat" tube is $\tilde{Z}=\phi_{f} Z=12.5$.

To assess the effect of this CR environment on chain relaxation, we examine the correlation function of the end to end vector $\mathbf{R}(t)$ of the chains

$$
\Phi(t)=\langle\mathbf{R}(t) \cdot \mathbf{R}(0)\rangle /\langle\mathbf{R} \cdot \mathbf{R}\rangle,
$$

which we calculate in the simulations using the multitau correlator of Ramirez et al. [25]. $\Phi(t)$ is, to first approximation, a measure of the "tube survival fraction" $\mu(t)$ since the endto-end vector of the chain at $\mathbf{R}(t)$ at time $t$ is correlated with $\mathbf{R}(0)$ only by the section of initial tube which remains occupied at time $t$. In fact, the quantity $\Phi(t)$ also determines the dielectric relaxation function of polymers (such as polyisoprene) with type-A dipoles, and this has been exploited to measure $\mu(t)$ for many entangled polymers $[26,27]$. The only difference between $\Phi(t)$ and $\mu(t)$ is an additional relaxation due to local fluctuations of the chain ends within the tube, whose contribution we consider below.

\section{PRESENTATION AND ANALYSIS OF SIMULATION DATA}

\section{A. Scaling analysis of the data}

In the absence of CR, CLF occurs via Rouse motion of the chain along the thin tube contour, relaxing a fraction of the chain $Z^{-1 / 2}$ in the Rouse time $\tau_{\mathrm{R}}=N^{2} \zeta b^{2} / 3 \pi^{2} k_{\mathrm{B}} T$. Hence, in the time regime where CLF dominates we expect $\Phi(t) \approx 1-C_{\mu} Z^{-1 / 2}\left(t / \tau_{\mathrm{R}}\right)^{1 / 4}$. Likhtman and McLeish [9] found that the constant $C_{\mu}=1.5$. The quarter power of time in this expression arises from the expected sub-Fickian scaling of chain end displacement within the Rouse model, as discussed in more detail in Sec. III B. Within our stochastic simulations, the constant first term of $\Phi(t)$ is subject to sampling fluctuations, which can be eliminated by examining the time derivative $d \Phi / d t$. In Fig. 3, we present the simulation data by plotting $-t^{3 / 4}(\mathrm{~d} \Phi / \mathrm{dt})$ versus simulation time, $t$, on a double logarithmic plot for a range of simulations, specifically:

(1) a simulation in which all CR is switched off,

(2) a simulation in which all the "fast" constraints $(90 \%$ of the total) are simply deleted (equivalent to $\tau_{C R}=0$ ), and

(3) simulations for a range of intermediate CR times, 3 $\leq \tau_{\mathrm{CR}} \leq 30000$ applied to the $90 \%$ of the slip-springs undergoing $\mathrm{CR}$.

Plotting the quantity $-t^{3 / 4}(\mathrm{~d} \Phi / \mathrm{dt})$ conveniently collapses the data across 7 orders of magnitude of time to a vertical range within a factor of 4 . It also ensures that regions of the data with the expected quarter-power time scaling for CLF appear as horizontal lines on the graph. CLF in the thin-tube

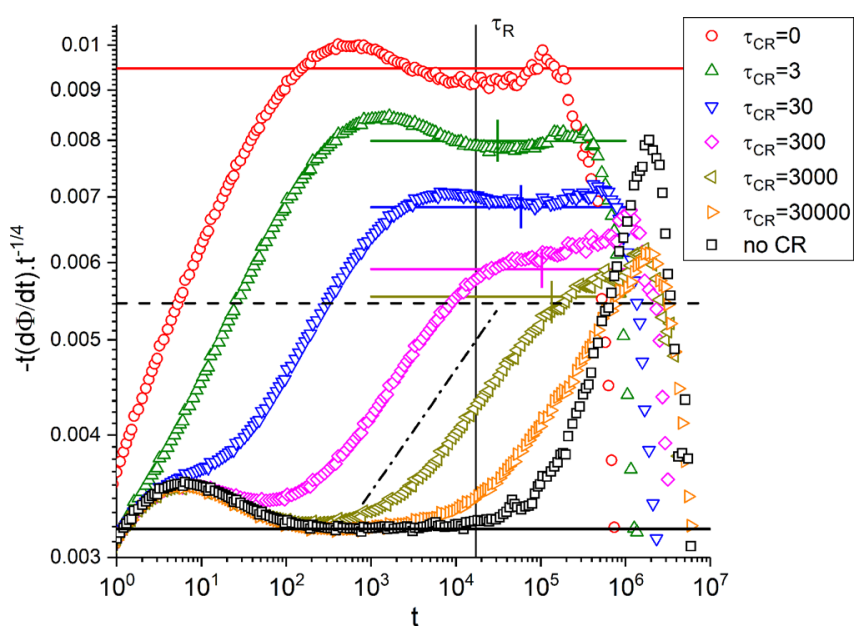

FIG. 3. $-t^{\frac{3}{4}}(\mathrm{~d} \Phi / \mathrm{dt})$ versus simulation time, $t$, for $f=0.1$ and for a range of $\mathrm{CR}$ times, $\tau_{\mathrm{CR}}$. All other simulation parameters given in the main text. Data for no $\mathrm{CR}$ correspond to motion purely in the thin tube, while data for $\tau_{\mathrm{CR}}=0$ correspond to motion purely in the fat tube. Also shown are horizontal lines corresponding to predicted CLF plateaus [as given by Eq. (A7)], and short vertical lines corresponding to predicted effective CLF times [as given by Eq. (A8)]. The lower solid horizontal line shows the CLF plateau for motion along the thin tube without CR. The horizontal dashed line corresponds to CLF along the fat tube in the limit of slow CR, with CLF time $\tau_{\mathrm{R}} / \phi_{f}$ giving $\Phi(t) \approx a-C_{\mu} \phi_{f}^{-1 / 4} Z^{-1 / 2}\left(t / \tau_{\mathrm{R}}\right)^{1 / 4}$, i.e., a CLF plateau a factor $\phi^{-1 / 4}$ higher than the thin tube CLF plateau. The full vertical line is the chain Rouse time, $\tau_{\mathrm{R}}$. The dash-dot line has a slope of $\frac{1}{8}$, as a guide to the eye, indicating the scaling predicted by Read et al. [11] when both CR and CLF are active concurrently. 
is therefore visible as the lower plateau between $t=10^{2}$ and $t=10^{4}$ for the simulation with no CR. A similar representation of the data, for the tube survival probability $\mu(t)$, was used previously by Likhtman and McLeish [9]. We note in passing that, at the very earliest times (not shown in Fig. 3), all the simulation data collapse onto the same curve for local diffusive motion of the connected beads. Here, we focus on the later time data relevant to entanglement effects.

In fact, the data for "no-CR" in Fig. 3 are extremely similar to the tube survival function from a one dimensional simulation presented by Likhtman and McLeish [9]. The CLF plateau begins at $t=10^{2}$, after a significant transient related to the establishment of effectively $1 \mathrm{D}$ motion along the tube contour in our 3D simulation. The CLF plateau ends at roughly the chain Rouse time $\tau_{R}$ (indicated by the vertical line close to at $t=10^{4}$ ) after which there is a strong peak due to reptation of well-entangled chains. So, for $t>\tau_{\mathrm{R}}$, the data grow with a slope of $1 / 4$ in Fig. 3, corresponding to one dimensional Fickian diffusion (in which distance traveled by the chain center of mass scales as $\sim t^{1 / 2}$, so that $\Phi(t) \approx 1-B t^{1 / 2}$ ). The data, as plotted in Fig. 3, then reach a peak just prior to the terminal reptation time.

A complication in analysing these data arises from the discovery [21] that the slip-springs themselves add significant friction to the along tube motion. This friction contribution is only partially mitigated by increasing the rate of slip-spring hopping between beads, because part of the time for a slipspring "hop" arises from waiting for an adjacent bead to enter the local vicinity of the slip-spring. The slip-spring friction should most likely be viewed as an artifact of the model, rather than something which corresponds to a physical phenomenon in real polymers. It is, however, necessary to account for the effect of this friction in understanding the results of the slip-spring model, and especially in mapping from the slip-spring model to the tube model. To fit the CLF plateau, we may assume the relation [9] $\Phi(t) \approx 1-C_{\mu} Z^{-1 / 2}\left(t / \tau_{\mathrm{R}}\right)^{1 / 4}$ with $C_{\mu}=1.5$ and then adjust the assumed friction constant as $\tau_{\mathrm{R}}=N^{2}\left(\zeta+\zeta_{\mathrm{sL}}\right) b^{2} / 3 \pi^{2} k_{\mathrm{B}} T$, where $\zeta_{\mathrm{SL}}$ is the effective friction for along-tube motion per polymer bead coming from the slip-spring friction. Using a value $\zeta_{\mathrm{SL}}=0.93$ in the no-CR simulations gives the horizontal black line in Fig. 3. We use this value in all subsequent analysis. However, for clarity of presentation we detail the effect of slip-spring friction on the presented equations in Appendix A, following the derivations in [21]. We note that it is also possible to fix $\zeta_{\mathrm{SL}}$ and adjust $C_{\mu}$ within a small range to fit the data; this makes little difference to our present analysis since the effects of adjusting $\zeta_{\mathrm{SL}}$ and $C_{\mu}$ largely cancel. The Rouse time $\tau_{R}$ indicated in Fig. 3 includes the slip-spring friction. Overall, the no-CR data are exactly as expected for monodisperse polymers in the absence of CR.

In Fig. 3, we observe that $\mathrm{CR}$ produces an acceleration of CLF, due to the increased freedom of the chain, and acceleration of the terminal relaxation. After sufficient CR has taken place, the chain explores a fat tube defined by only the slow slip-spring constraints. The extreme case of $\tau_{\mathrm{CR}}=0$ (i.e., free, unimpeded chain motion along the fat tube) exhibits a short CLF plateau, visible at times of order $10^{4}$, followed by a much weaker reptation peak associated with a smaller number of entanglements $\left(\tilde{Z}=\phi_{f} Z=12.5\right)$. In their equivalent plots for tube survival fraction $\mu(t)$ of monodisperse chains, Likhtman and McLeish [9] observed a weakening of the peak for final reptation as the number of entanglements was reduced. Comparing the plots for $\tau_{\mathrm{CR}}=0$ and the data with no CR, we note that the end of the CLF plateau is at a comparable simulation time for both sets of data, i.e., around the chain Rouse time indicated by the long vertical line. (The apparent end of the CLF plateau for the $\tau_{\mathrm{CR}}=0$ data appears to be a factor of 3 later than the Rouse time, most likely due to the terminal peak being weak for the small number of slow fat tube entanglements.) We estimate the height of the CLF plateau for the $\tau_{\mathrm{CR}}=0$ data by noting that a fraction of the chain $\tilde{Z}^{-1 / 2}=\left(\phi_{f} Z\right)^{-1 / 2}$ relaxes in the Rouse time $\tau_{\mathrm{R}}$. Hence, we expect $\Phi(t) \approx a-C_{\mu} \phi_{f}^{-1 / 2} Z^{-1 / 2}\left(t / \tau_{\mathrm{R}}\right)^{1 / 4}$, i.e., we expect the plateau to be a factor $\phi_{f}^{-1 / 2}$ higher than for the thin tube. We show the expected plateau value via the upper red horizontal line in Fig. 3 (which has been corrected to account for slip-spring friction). The predicted value is within $5 \%$ of the plateau in the data.

Nonzero CR times produce intermediate behavior between the no-CR and $\tau_{\mathrm{CR}}=0$ data. At times of order $\tau_{\mathrm{CR}}$ the data depart from the no-CR data and increase toward an upper CLF plateau which is visibly established for $\tau_{\mathrm{CR}}=3$ and 30 , and approached for $\tau_{\mathrm{CR}}=300$ and 3000 . We interpret this plateau as indicating CLF in the fat tube, but at a slower rate than for $\tau_{\mathrm{CR}}=0$. The plateau also persists to later times than for the $\tau_{\mathrm{CR}}=0$ data, before exhibiting a reptation peak. This peak is similar in shape to the $\tau_{\mathrm{CR}}=0$ data, but also at a delayed time. These observations suggest that the chain is effectively moving as though along the fat tube for both CLF and reptation, but with an increased effective friction. Before attempting to predict this effective friction theoretically, we can test whether such a description is plausible by manually rescaling time, $t$, in both axes of the plot, where the scaling factor $\alpha$ is chosen for each $\tau_{\mathrm{CR}}$ so as to overlap the data. Here, we are testing whether a single rescaling factor, $\alpha$, can achieve a data overlap in the two separate (independent) dimensions of the plot. This rescaling is done in Fig. 4. The rescaling of time gives an excellent overlap of the data for $\tau_{\mathrm{CR}}=0,3$, and 30 , matching the CLF plateau and terminal peak to within simulation noise. It also gives a close overlap for $\tau_{\mathrm{CR}}=300$ and 3000. This overlap of data confirms that the effective chain motion on large length scales is self-similar across the range of CR times, which implies that the motion can be coarsegrained to an effective chain motion along the fat tube.

In the limit of slow $\tau_{\mathrm{CR}}$ (while $\mathrm{CR}$ remains fast enough to locally explore the fat tube), Read et al. [11,28] predict that the chain transport along the thin tube alone is sufficient to give a renormalized reptation and CLF along the fat tube. The tortuous path of the thin tube gives rise to a larger effective friction along the smoother fat tube, resulting in an enhanced CLF time of $\tau_{\mathrm{R}} / \phi_{f}$. We expect a fraction of the chain $\tilde{Z}^{-1 / 2}=\left(\phi_{f} Z\right)^{-1 / 2}$ to relax in this time, so that $\Phi(t) \approx a-C_{\mu} \phi_{f}^{-1 / 4} Z^{-1 / 2}\left(t / \tau_{\mathrm{R}}\right)^{1 / 4}$, i.e., for slow $\mathrm{CR}$ we expect a CLF plateau a factor $\phi^{-1 / 4}$ higher than the thin tube CLF plateau: This value is represented by the dashed horizontal line in Fig. 3. In practice, all the data which exhibit a fat tube CLF plateau lie between the red and the dashed 


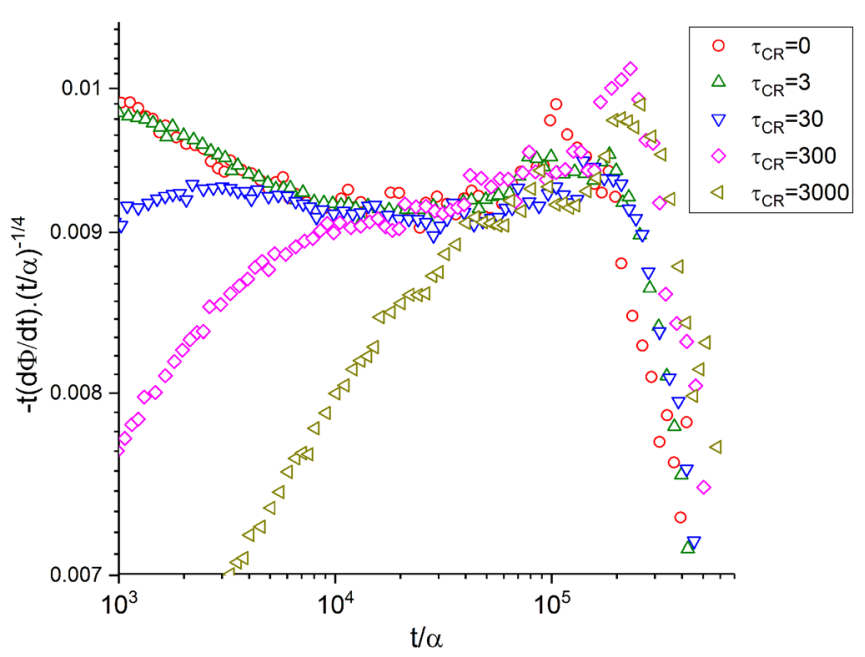

FIG. 4. Selected data from Fig. 3 replotted with the time rescaled by a factor $\alpha$, i.e., we plot $-t^{3 / 4}(\mathrm{~d} \Phi / \mathrm{dt}) \alpha^{1 / 4}$ versus $t / \alpha$. Values of $\alpha$ used are $1,1.85,3.0$, 5.0 , and 6.5 for CR times $\tau_{\mathrm{CR}}=0,3,30,300$, and 3000, respectively.

horizontal lines. This is because CR itself provides an additional, and accelerating, mechanism for chain motion (both CLF and reptation) along the fat tube, which should be added to diffusion along the thin tube. Read et al. [11] predict the effective friction per bead for motion along the fat tube to be

$$
\frac{1}{\zeta_{\text {fat }}}=\frac{\phi_{f}}{\zeta}+\frac{1}{\zeta_{\mathrm{CR}}+\frac{\zeta}{1-\phi_{f}}} .
$$

The first term on the right accounts for chain motion along the thin tube, projected onto the fat tube contour, and is the only contribution in the limit of slow $\mathrm{CR}\left(\zeta_{\mathrm{CR}} \rightarrow \infty\right)$. The second term accounts for motion along the fat tube due to $\mathrm{CR}$, where $\zeta_{\mathrm{CR}} \propto \tau_{\mathrm{CR}}$ is the effective friction per bead from $\mathrm{CR}$ events. In the limit $\zeta_{\mathrm{CR}} \rightarrow 0$, monomer friction again dominates and $\zeta_{\text {fat }} \rightarrow \zeta$. We note that the prediction by Read et al. $[11,28]$ of an enhanced CLF time of $\tau_{\mathrm{R}} / \phi_{f}$ applies only in the regime of slow CR, where $\zeta_{\mathrm{CR}}$ is large and the first term on the right hand side of Eq. (2) is the dominant one. For faster CR, the second term starts to contribute, and the CLF time is accelerated.

Shivokhin et al. [21] present corrections to Eq. (2) for the slip-spring model, including the slip-spring friction, dilution effects, and an improved crossover to monomeric friction for fast CR. We summarize their results in Appendix A and show how they may be used to predict the expected CLF plateau [Eq. (A7)], and CLF relaxation time for each simulation [Eq. (A8)]. These results are indicated by the horizontal and vertical lines, respectively, in Fig. 3 for each value of $\tau_{\mathrm{CR}}$. The plateau levels are accurately predicted. As with the data for $\tau_{\mathrm{CR}}=0$ described above, for each nonzero CR time the apparent end of the CLF plateau is consistently a factor of 3 later than the predicted CLF time. Consequently, the horizontal shift of the data, and in particular, the crossover to terminal reptation dynamics, follows the horizontal shift in the predicted CLF relaxation time as $\tau_{\mathrm{CR}}$ is varied.

In between the early time plateau (CLF in thin tube) and late time plateau (CLF in fat tube) is a regime where both
CR and CLF are active concurrently. In this case, the chain may be expected to explore an effective supertube represented by dilution $\phi \sim t^{-1 / 2}$ from CR Rouse motion. In this regime, $\operatorname{Read}$ et al. [11] predict $\Phi(t) \approx a-C t^{-3 / 8}$, a scaling which is indicated by the dash-dot line of slope $\frac{1}{8}$ in Fig. 3 . While this initially appears to give a plausible prediction of the slope in this intermediate region, especially for the slowest $\tau_{\mathrm{CR}}$, there is actually not enough separation between the different time regimes to establish this intermediate scaling. In particular, contributions to $\Phi(t)$ from tube dilution contribute significantly to the data, and it is likely that the $\phi \sim t^{-1 / 2}$ scaling for CR Rouse motion is not fully established, as we now discuss.

\section{B. Calculation of full time dependence of the data}

We now aim to describe the full time-dependence of the simulation data. The sub-Fickian monomer displacement $x \sim t^{-1 / 4}$ within the Rouse model can be understood from a simple scaling argument $[29,30]$ assuming an effective drag constant based on how many beads in a subsection of chain move coherently at a given timescale. The number, $n$, of beads moving coherently can be obtained by equating current timescale $t$ with the Rouse time $\tau_{\mathrm{R}} \sim n^{2} \zeta b^{2} / k_{\mathrm{B}} T$ for those $n$ monomers, giving $n(t) \sim \sqrt{t k_{\mathrm{B}} T / \zeta b^{2}}$. These beads then move with diffusion constant $D \sim k_{\mathrm{B}} T / n \zeta$, so that on timescale $t$ they are expected to diffuse a mean square distance $x^{2} \sim D t \sim \sqrt{t k_{\mathrm{B}} T b^{2} / \zeta}$, which is also (self consistently) equal to the typical chain size $x^{2} \sim n b^{2}$ for a chain with $n$ beads. Hence, the sub-Fickian diffusion is understood to arise from an effective drag coefficient which increases on increasing length- or time-scales for the chain motion.

We may specialize the above scaling argument to deal with the particular case of a chain end undergoing CLFs in a fat tube (whose effective diameter may increase with time due to $\mathrm{CR}$ ). We are required to obtain the effective diffusion distance $l_{\text {fat }}$ of the chain end along the diluted fat tube, and we note that the friction constant per monomer for this motion is $\zeta_{\text {fat }}$, as obtained from Eq. (2). Hence, we anticipate $\left(\mathrm{d} l_{\text {fat }}^{2} / \mathrm{d} t\right) \propto 1 / n(t) \zeta_{\text {fat }}$ where $n(t)$ is the number of monomers moving coherently with the chain end by Rouse motion, obtained by inverting the Rouse relaxation time, $t \sim$ $n^{2} \zeta_{\text {fat }} b^{2} / k_{\mathrm{B}} T$ for $n$ beads, to give $n(t) \sim \sqrt{t k_{\mathrm{B}} T / \zeta_{\text {fat }} b^{2}}$. We then note that moving a distance $l_{\text {fat }}$ along the fat tube corresponds to relaxing a fraction $\tilde{\Phi}_{\mathrm{CLF}}=l_{\mathrm{fat}} / a_{\mathrm{fat}} Z_{\mathrm{fat}}=$ $l_{\text {fat }} / a Z \phi^{1 / 2}$ of the total tube length by CLFs (the factor $\phi^{1 / 2}$ assumes a dilution exponent of 1$)$. We would anticipate $\Phi(t)=1-\tilde{\Phi}_{\mathrm{CLF}}$. We may write $\mathrm{d} \tilde{\Phi}_{\mathrm{CLF}}^{2}=\mathrm{d} l_{\text {fat }}^{2} / a^{2} Z^{2} \phi$, which yields

$$
\frac{\mathrm{d} \tilde{\Phi}_{\mathrm{CLF}}^{2}}{\mathrm{~d} t}=\frac{C_{\mu}^{2}}{2 Z^{2}}\left(\frac{3 \pi^{2} k_{\mathrm{B}} T}{\phi^{2} N_{e}^{2} b^{2} \zeta_{\mathrm{fat}} t}\right)^{\frac{1}{2}} .
$$

In the limit of pure CLF in the thin tube, $\phi=1$ and $\zeta_{\text {fat }}=\zeta$, so Eq. (3) can be written as

$$
\frac{\mathrm{d} \tilde{\Phi}_{\mathrm{CLF}}^{2}}{\mathrm{~d} t}=\frac{C_{\mu}^{2}}{2 Z^{2}}\left(\frac{1}{\tau_{\mathrm{e}} t}\right)^{\frac{1}{2}}=\frac{C_{\mu}^{2}}{2 Z}\left(\frac{1}{\tau_{\mathrm{R}} t}\right)^{\frac{1}{2}}
$$


which has solution $\tilde{\Phi}_{\mathrm{CLF}}=C_{\mu} Z^{-1 / 2}\left(t / \tau_{\mathrm{R}}\right)^{1 / 4}$, consistent with the formula $\Phi(t) \approx 1-C_{\mu} Z^{-1 / 2}\left(t / \tau_{\mathrm{R}}\right)^{1 / 4}$ used to determine the thin tube CLF plateau in Fig. 3, discussed above. The proportionality constant in Eq. (3) has been fixed to ensure this consistency, and the equation then automatically predicts the corresponding fat tube plateaus obtained in Eq. (A7).

The chain is initially constrained in the thin tube with dilution $\phi=1$, but CR allows the chain to explore a gradually widening volume, parameterized by a Rouse scaling $\phi=\left(t / \tau_{\text {hop }}\right)^{-1 / 2}$ with CR hop time $\tau_{\text {hop }}=c_{\phi}\left(N_{e}^{2} b^{2} \zeta_{\mathrm{CR}} / k_{\mathrm{B}} T\right)$ until the effective dilution approaches the fat tube $\phi=\phi_{f}$ (here, $c_{\phi}$ is a scaling constant related to the rate of local equilibration due to $\mathrm{CR}$ events). To ensure smooth transitions between these three regimes, we employ a crossover function

$$
\phi=\left(\phi_{f}^{b}+\frac{1}{\left[\left(1-\phi_{f}^{b}\right)^{-\frac{a}{b}}+\left(t / \tau_{\mathrm{hop}}\right)^{\frac{a}{2}}\right]^{\frac{b}{a}}}\right)^{\frac{1}{b}}
$$

where $a$ controls the smoothness of the early time crossover, and $b$ the late time crossover. Employing this in Eq. (3), and in Eq. (2) with $\phi_{f}$ replaced by $\phi$, allows $\tilde{\Phi}_{\mathrm{CLF}}(t)$ to be obtained by numerical integration. In practice, we use a modified expression for $\zeta_{\text {fat }}$, which includes the effects of slipspring friction, as detailed in Appendix A.

Finally, we note that since the function $\Phi(t)$ [as given in Eq. (1)] is based on correlations in fluctuations of the end-toend vector of the chain, it is not exactly equal to the tube survival function, $\mu(t)$, but is additionally affected by fluctuations of the chain about the tube axis at the ends of the surviving tube. Watanabe et al. [27] noted a similar issue in discussing dielectric relaxation of star chains. It is therefore necessary to include a further correction for this effect. A fluctuation of mean square amplitude $\left\langle\Delta r_{e}^{2}\right\rangle$ of the chain, independently applied at both ends of a tube section, gives rise to correlations in the end to end vector of form

$$
\langle\mathbf{R}(t) \cdot \mathbf{R}(0)\rangle=\langle\mathbf{R}(0) \cdot \mathbf{R}(0)\rangle-\left\langle\Delta r_{e}^{2}\right\rangle .
$$

Assuming $\left\langle\Delta r_{e}^{2}\right\rangle=c_{\text {ends }} N_{e} b^{2} / \phi$ (i.e., fluctuations of order the diluted tube diameter with proportionality constant $c_{\text {ends }}$ ), and including also the effects on the tube survival function due to CLFs in Eq. (1), gives

$$
\Phi(t)=1-\tilde{\Phi}_{\mathrm{CLF}}(t)-\tilde{\Phi}_{\mathrm{ends}},
$$

where $\tilde{\Phi}_{\text {ends }}=c_{\text {ends }} / \phi Z$.

Evidently, in the above discussion, there are several, in principle model-dependent, fitting parameters, yet reasonable choices $c_{\phi}=c_{\text {ends }}=1, a=2$, and $b=1$ give an excellent description of the data for slow $\mathrm{CR}, 300 \leq \tau_{\mathrm{CR}} \leq 30000$, as shown in Fig. 5. These predictions use the above expressions, modified for inclusion of slip-spring friction as detailed in Appendix A. The data for faster CR are affected by the early time transient exhibited by all the data for $t \leq 10^{2}$, so it was not possible to match those data using the same parameters:
This evidently affects our ability to predict the data for $\tau_{\mathrm{CR}}$ $\leq 30$ for which the theory does not match the data quantitatively. Figure 5 also illustrates the non-negligible contribution from fluctuations of the chain about the tube axis, as parameterized by $\tilde{\Phi}_{\text {ends }}$ : The dashed lines indicate the predictions if this component is not included (it is possible to match the data by setting $\tilde{\Phi}_{\text {ends }}=0$ and adjusting other parameters to compensate, but we consider the parameters chosen are physically reasonable). With the same set of parameters, we are also able to match with similar accuracy simulation data for $f=0$, i.e., in which CR is applied to all slip-springs, as shown on the right of Fig. 5. Again prediction of the data is excellent for $300 \leq \tau_{\mathrm{CR}} \leq 30000$, while data for $\tau_{\mathrm{CR}} \leq 30$ are not quantitatively predicted by the model.

In summary, in this section we have constructed a model, based on a scaling argument for Rouse dynamics of the chain ends, for CLFs in a tube which is gradually widening (being diluted) due to CR events. For sufficiently slow CR, the resulting predictions (using reasonable parameterization) are in close agreement with the simulation data as shown in Fig. 5. It seems possible that a theoretical treatment along the lines developed in this section could provide the basis for a description of CLF in more general CR environments with multiple CR times.

\section{IMPLICATIONS FOR RHEOLOGY OF BINARY POLYMER MIXTURES: REDRAWING THE VIOVY DIAGRAM}

To the extent that the slip-spring model is a realistic predictor of the dynamics of entangled polymers, the above analysis indicates the following conclusions: (i) That CR in a binary mixture provides a mechanism for accelerated CLFs; (ii) that this can be considered in terms of motion along a diluted fat tube, with an appropriately rescaled effective friction constant; and (iii) that this effective friction arises from a combination of chain motion along the thin tube and CR-activated motion along the fatter tube. The expressions for friction derived by Read et al. [11], as given in Eq. (2), appropriately combine these two contributions, so that we are able to account for the observed plateaus for CLF in the fat tube as observed in our slip-spring simulations. We are also able to describe the transient behavior as described in Sec. III B.

It is also the case that CLF in the fat tube, when it occurs, provides a mechanism for accelerating the terminal relaxation of the chains, because it shortens the distance required for the chain to reptate in order to complete the relaxation process. This has practical implications for the rheology of both binary and polydisperse polymer mixtures. In order to illustrate this, we now revisit the diagram created by Viovy et al. for binary blends [18], but reconsider it in the light of CLFs of both short and long polymers.

\section{A. Construction of the diagram}

\section{General description of the modified diagram}

For monodisperse entangled polymers, with $Z$ entanglements, the reptation relaxation time including the effect of CLF can in general be written in the form 

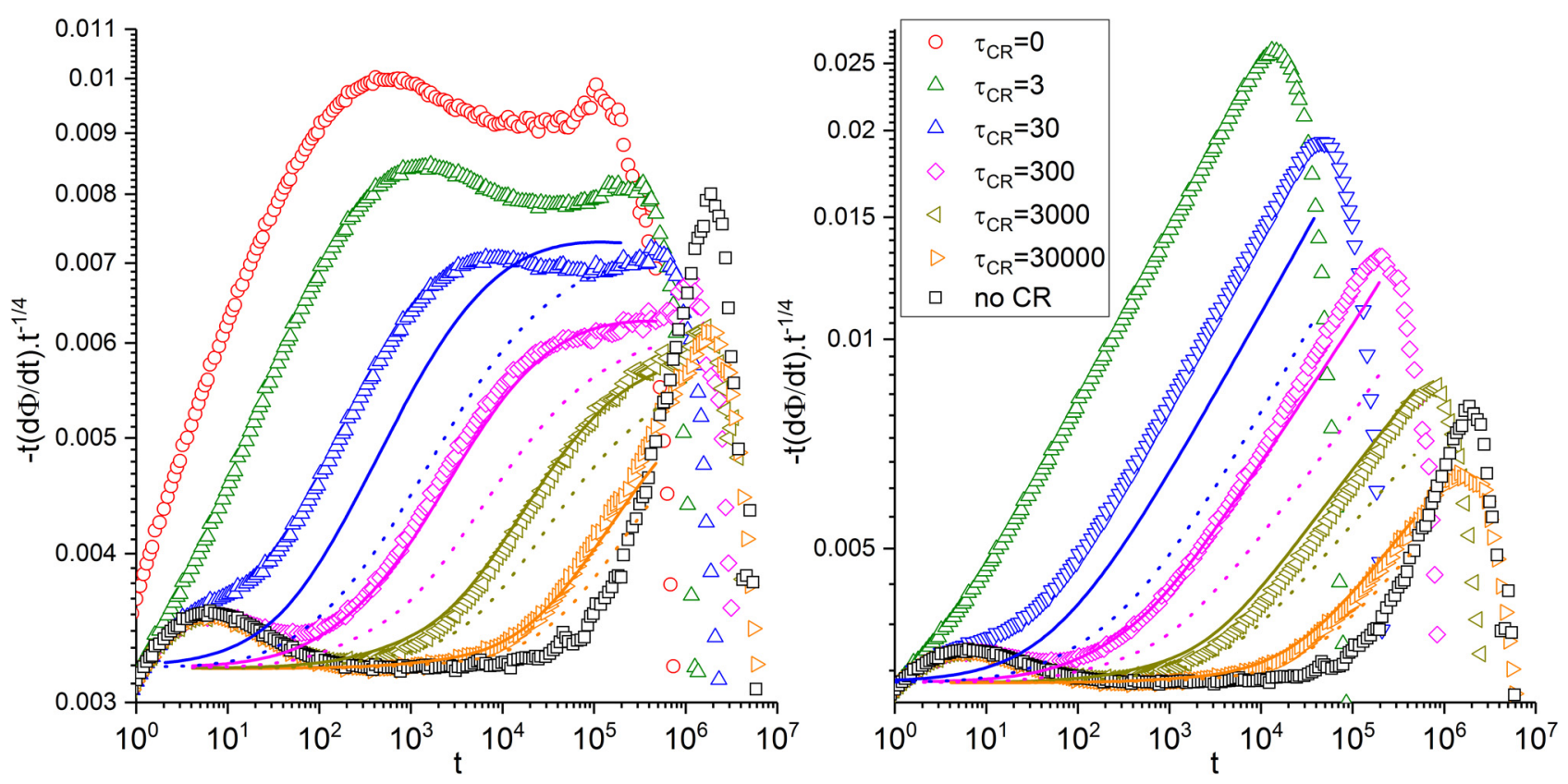

FIG. 5. Left: simulation data from Fig. 3 together with theoretical predictions of the data using the model described in Sec. III B. Solid lines present predictions including fluctuations of the chain around the tube axis, while dashed lines indicate predictions with $\tilde{\Phi}_{\text {ends }}=0$ and other parameters fixed. Right: simulation data and theoretical predictions for $f=0$, i.e., in which $\mathrm{CR}$ is applied to all slip-springs, with all other parameters identical to those in Fig. 3. Data for no CR correspond to motion purely in the thin tube; these data are identical for both plots. Data for $\tau_{\mathrm{CR}}=0$ correspond to motion purely in the fat tube.

$$
\tau_{\mathrm{d}}=3 Z^{3} f(Z) \tau_{\mathrm{e}}
$$

where $\tau_{\mathrm{e}}$ is the entanglement time, and the function $f(Z)$ incorporates the correction to pure reptation from CLF. Various functional forms for $f(Z)$ have been proposed (for example, the apparent 3.4 power law for viscosity versus molecular weight could be incorporated using $f(Z) \sim Z^{0.4}$ ). From fits to their simulation data, McLeish and Likhtman obtained the functional form

$$
f(Z)=1-\frac{3.38}{\sqrt{Z}}+\frac{4.17}{Z}-\frac{1.55}{Z^{1.5}},
$$

which we shall use in this work (in fitting to slip-spring data in a previous publication [21], we found that replacing 1.55 with 1.4 in the last term gave a better representation of the data, but the difference is marginal for the present discussion). The typical $\mathrm{CR}$ time from the short chains with $Z_{\mathrm{S}}$ entanglements in a binary mixture is therefore equal to the reptation time of the short chains, corrected for CLF

$$
\tau_{\mathrm{CR}}=\tau_{\mathrm{dS}}=3 Z_{\mathrm{S}}^{3} f\left(Z_{\mathrm{S}}\right) \tau_{\mathrm{e}}
$$

The form of this suggests that, instead of the usual Graessley parameter, $G r=Z_{\mathrm{L}} / Z_{\mathrm{S}}^{3}$, in which the $Z_{\mathrm{S}}^{3}$ indicates the scaling of CR time with $Z_{S}$, we should use a modified Graessley parameter which we propose should take the form

$$
G r^{*}=\frac{Z_{\mathrm{L}}}{3 Z_{\mathrm{S}}^{3} f\left(Z_{\mathrm{S}}\right)}=\frac{Z_{\mathrm{L}} \tau_{\mathrm{e}}}{\tau_{\mathrm{CR}}} .
$$

There is an argument for also considering CLF of the long chains directly in our definition of a modified Graessley parameter, but since the degree of long chain CLF is strongly dependent on composition (with potential for CLF in thin or fat tubes), it turns out to be better to consider the effects of long chain CLF separately, during the construction of the diagram, as we do below.

For the horizontal axis of our modified Viovy diagram, we retain the variable $\tilde{Z}_{\mathrm{L}}=\phi_{f} Z_{\mathrm{L}}$, the number of entanglements along the diluted "fat tube" for the long chains, under the assumption of dilution exponent of 1 . Hence, our modified diagram takes the form of a "map" in the two-dimensional plane of co-ordinates $\left(\tilde{Z}_{\mathrm{L}}, G r^{*}\right)$ and is presented in Fig. 6.

As with the original Viovy diagram, shown schematically in Fig. 1, there are numerous lines which divide the diagram into a number of regions of the parameter space, where the qualitatively different binary blend behavior may be expected. These lines follow the overall geometry of the original diagram deduced by Viovy et al., but augment it with the effects of CLF. Some lines are also shifted in position from the original diagram, by consideration of the numerical results from our slip-spring simulations.

We first discuss the various numbered regions labeled in Fig. 6, before detailing the construction of the lines in Sec. IV A 2. The numbers 1-4 refer to regions of the original Viovy diagram as shown in Fig. 1, while the letters a, b, c refer to subdivisions of those regions due to CLF effects.

- In region 1 of the diagram, the dilute long chains fully relax via CR Rouse motion.

- In region 2 of the diagram, the CR Rouse motion is terminated by reptation of the long chains. In region 2a, reptation is not at all accelerated by $\mathrm{CR}$, while in region $2 \mathrm{~b}, \mathrm{CR}$ leads to enhanced CLF in a partially dilated tube speeding up the terminal reptation.

- In region 3 of the diagram, the chain is locally able to explore the fat tube before terminal reptation, so the 

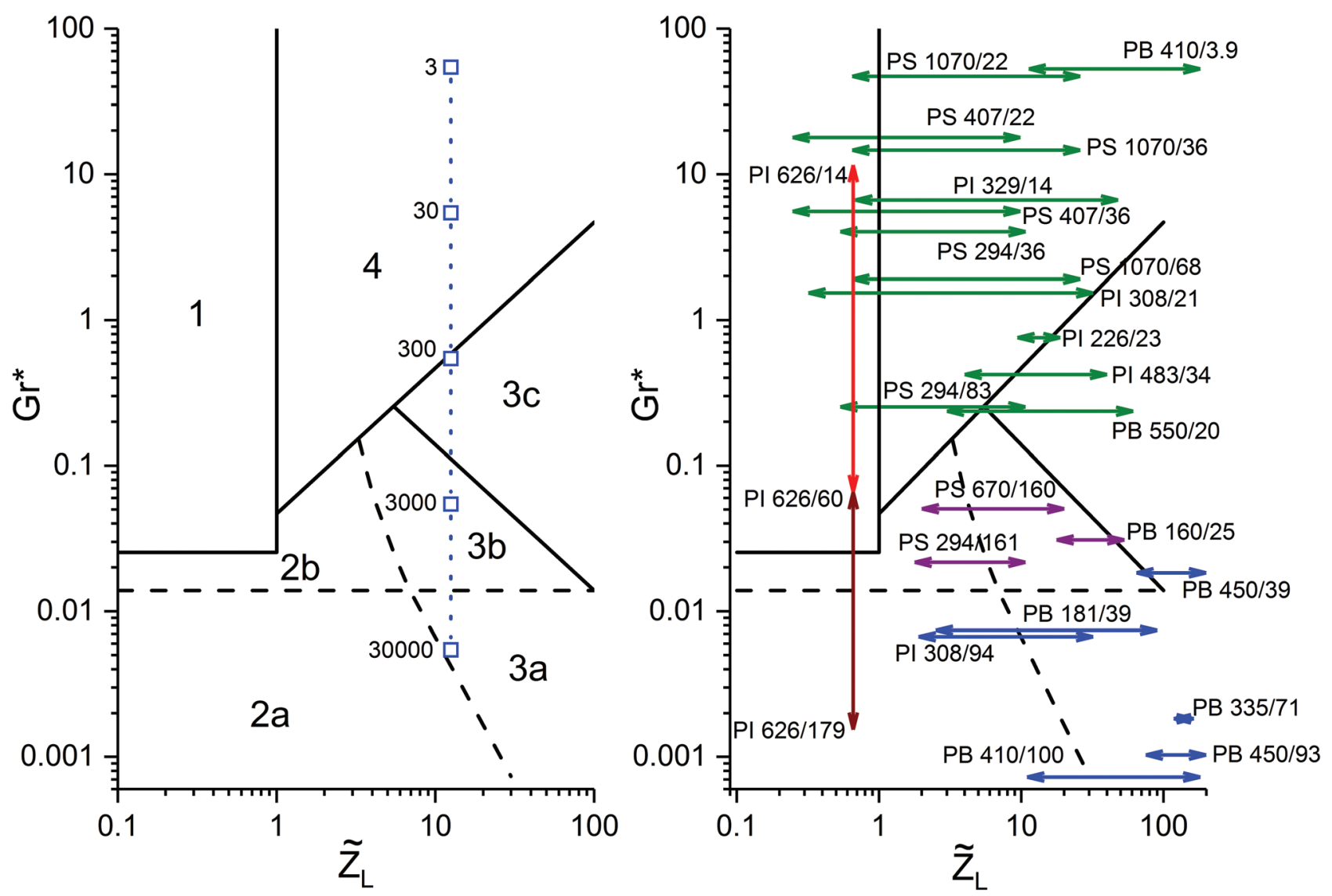

FIG. 6. Left: construction of the modified Viovy diagram, with regions of the diagram labeled as described in the main text. The blue squares, and vertical dashed line, indicate the location of our slip-spring simulations on the modified diagram for different values of $\tau_{\mathrm{CR}}$. Right: Location of a substantial set of literature blends on the diagram. Arrows indicate the range covered by the blends for these data, but do not extend to the value of $Z_{\mathrm{L}}$ for the pure long chain component. Green arrows indicate blends where it is very clear that relaxation of long chains is accelerated by dilution with shorter chains. Blue arrows indicate blends where the long chain relaxation is clearly not accelerated by shorter chains. Purple arrows indicate data where there may be some acceleration of terminal relaxation when long chains are diluted by shorter ones. To the left of the diagram, the red and brown vertical arrows indicate a set of data in which dilute long chains are measure in matrices of different short chain molecular weight. The red arrow indicates data which clearly follow CR Rouse scaling of terminal relaxation, and the brown arrow indicates data which depart from this). Blends are labeled according to material (PolyIsoprene, PolyButadiene or PolyStyrene) and molecular weight of the long/short component in $\mathrm{kg} / \mathrm{mol}$. References for the data are given in Table I.

terminal modulus is set by entanglements between long chains. However, the dominant process governing chain transport along the fat tube is via chain motion along the (more tortuous) thin tube. In region 3a, reptation is not at all accelerated by CR [giving Eq. (19) below]; in region $3 \mathrm{~b}, \mathrm{CR}$ leads to enhanced CLF in a partially dilated tube [accelerating the terminal relaxation as in Eq. (21)]; in region 3c, CR leads to enhanced CLF in a fully dilated fat tube [accelerating the terminal relaxation as in Eq. (20)].

- In region 4 of the diagram, $\mathrm{CR}$ of the thin tube along the fat tube is faster than chain motion along the thin tube. Hence, the chain undergoes both CLF and reptation in the fat tube at an accelerated rate set by the CR motion with effective friction constant per monomer given by Eq. (2).

Importantly, we have identified new regions of the diagram ( $3 \mathrm{~b}$ and $3 \mathrm{c}$ ) in which CLF in a partially or fully dilated tube may be expected to lead to accelerated relaxation of the long chains, even when the dominant chain transport is along the thin tube. In these regions, Viovy et al. [18] predicted no acceleration of the terminal relaxation, in contrast to our predictions, so this result is an important correction.

Before moving on to a discussion of how the lines on the diagram are constructed, we note one additional relevant factor for real polymeric liquids. It is often assumed that the effective friction for CR motion is simply proportional to the relaxation time of the short chains. This is certainly the case for slow CR, but as the CR time from short chains becomes faster, the local Rouse friction of the long chains becomes an additional factor which slows their CR Rouse relaxation. There is thus, toward the top of the Viovy diagram (in regions 1 and 4), a further crossover to motion dominated by local monomer friction rather than friction from CR events. However, as noted by Shivokhin et al. [21], this crossover is surprisingly broad. We discuss this feature in Subsection 1 of Appendix C, noting that in practice the crossover region extends to short chain lengths as long at 10 entanglements. Figure 6 is constructed without including the effects of this broad crossover; it is possible that the breadth of this crossover region may, in some practical cases, extend out toward the lines at the boundaries of regions 1 and 4, shifting their positions. 


\section{Details of construction of the lines on the diagram}

We now describe the construction of the modified Viovy diagram. We consider first the upper right hand side of the diagram, since this is the region most relevant to our above simulations. The long chains will entangle with other long chains provided $\tilde{Z}_{\mathrm{L}}>1$, and this gives a vertical line toward the top of the diagram in Fig. 6, separating dilute from concentrated long chains. This line was present in the original Viovy diagram.

A second line of the diagram is obtained by considering which of the two terms in Eq. (2) is dominant for the effective friction along the fat tube. For fast $\mathrm{CR}, \zeta_{\mathrm{CR}}$ is small and the second term dominates, so that motion directly along the fat tube (mediated by CR events) is the major process. For slow $\mathrm{CR}$, the chain can more readily move along the fat tube by snaking its way along the more tortuous contour of the thin tube (we note that motion along the thin tube also results in motion along the fat tube!). The balance between these two modes of motion can be obtained at a scaling level by comparing the bare reptation time in the thin tube $\left(3 Z_{\mathrm{L}}^{3} \tau_{\mathrm{e}}\right)$ with the reptation time for direct motion along the fat tube mediated by $\mathrm{CR}$ events, which scales as $\left(3 \tilde{Z}_{\mathrm{L}} Z_{\mathrm{L}}^{2} \tau_{\mathrm{CR}}\right)$. We find that the two processes are of similar magnitude when

$$
3 Z_{\mathrm{L}}^{3} \tau_{\mathrm{e}}=3 \tilde{Z}_{\mathrm{L}} Z_{\mathrm{L}}^{2} \tau_{\mathrm{CR}}
$$

which implies that

$$
G r^{*} \approx \tilde{Z}_{\mathrm{L}}
$$

An equivalent line to this was present on the original Viovy diagram. However, the scaling argument just presented ignores quite substantial prefactors, assumed to be "order 1" constants. It is more accurate to draw the line based upon the point where the two terms in Eq. (2) are of similar magnitude, using the results obtained from the slip-spring model (especially for the effective friction from CR events) to quantify this. This is done in Appendix B, and the result is that the line should in fact be drawn at

$$
G r^{*} \approx 0.047 \tilde{Z}_{\mathrm{L}}
$$

which substantially moves the crossover from that predicted by the scaling argument in Eq. (13). The main reason for this shift in the line is that relaxation due to $\mathrm{CR}$ is substantially faster than would be expected from simple scaling arguments, as discussed in Appendices B and C 1 .

The line predicted by Eq. (14) is shown separating regions 4 and $3 c$ in Fig. 6. Although drawing a line on a diagram seems to imply a sharp crossover, the data shown in Fig. 3 illustrate that, for polymers of realistic length, there is not a sharp boundary between the two regimes, and rather the transition from one to the other is somewhat smooth and continuous.

All the above assumes that it is appropriate to consider motion in the fat tube at all. However, for sufficiently slow $\mathrm{CR}$, it takes time for the thin tube to locally explore the fat tube, i.e., it takes finite time for equilibration of the thin tube over the size of a fat tube entanglement. If there are $\phi_{f}^{-1}$ thin tube segments per fat tube segment, then this equilibration time is the CR-Rouse time of $\phi_{f}^{-1}$ tube segments, which scales as

$$
\tau_{\mathrm{e}, \mathrm{f}} \approx \tau_{\mathrm{CR}} \phi_{f}^{-2}
$$

This time also corresponds to the long time crossover used in Eq. (5): We can use this equation to obtain a more accurate expression, consistent with the results of the slip-spring simulations. We demonstrate this in Appendix B, giving the result

$$
\tau_{e, f} \approx 1.39 \tau_{\mathrm{CR}} \phi_{f}^{-2}
$$

(In fact, the constant 1.39 could here and below be reasonably approximated as 1 for the purposes of the logarithmic plot of the Viovy diagram.) We may compare this timescale with other relaxation times to determine whether the fat tube is relevant to chain dynamics and stress relaxation at those timescales.

One such timescale corresponds to the transition from CLF to the onset of centre of mass reptation motion. According to tube theory, this transition occurs at the longest CLF time of the chain. For monodisperse chains in a fixed tube, this CLF time is just the Rouse time of the chain. However, if we consider motion in the fat tube, then the longest CLF time is the effective Rouse time using the rescaled friction from Eq. (2). If CR is sufficiently slow that the first term in Eq. (2) dominates, then this CLF time was found by Read et al. $[11,28]$ to be $\tau_{\mathrm{CLF}}=\tau_{\mathrm{R}} / \phi_{f}$, where $\tau_{\mathrm{R}}=Z_{\mathrm{L}}^{2} \tau_{\mathrm{e}}$ is the Rouse time of the long chains.

We may compare the fat tube CLF time $\tau_{\text {CLF }}$ to the fat tube equilibration time $\tau_{e, f}$. If $\tau_{\mathrm{CLF}}>\tau_{e, f}$, then the chain will have had opportunity to explore the fat tube locally before undergoing its deepest possible CLF motion in the fat tube. If this is the case we expect CLF in the fat tube to occur, and for this in turn to accelerate the terminal relaxation of the chain. On the other hand, if $\tau_{\mathrm{CLF}}<\tau_{e, f}$, then the transition to terminal reptation must occur before the chain has locally explored the fat tube, and so full CLF in the fat tube is no longer a possible mechanism for accelerating the terminal relaxation (although CLF in a partially dilated tube may still be possible). This can be seen in the simulation data in Fig. 3 where for $\tau_{\mathrm{CR}}=30000$ the plateau corresponding to CLF in the fat tube is not attained before the final transition to terminal reptation. Arguably, for $\tau_{\mathrm{CR}}=3000$ the fat tube CLF plateau is not fully attained either. Consequently, the line where $\tau_{\mathrm{CLF}}=\tau_{e, f}$ marks an important transition above which full CLF in the fat tube occurs. In terms of the Viovy diagram parameters, this gives

$$
G r^{*}=1.39 \tilde{Z}_{\mathrm{L}}^{-1}
$$

This is a new line on the diagram, not present in the original work of Viovy, because it concerns the effects of CLF. It is shown as the line separating regions $3 c$ and $3 b$ in Fig. 6. 
With our modified Graessley parameter from Eq. (11), the three lines obtained above $\left(\tilde{Z}_{\mathrm{L}}=1, G r^{*}=0.047 \tilde{Z}_{\mathrm{L}}\right.$ and $G r^{*}=1.39 \tilde{Z}_{\mathrm{L}}^{-1}$ ) are expected to be fixed lines in our modified Viovy diagram, i.e., they depend only on the composite parameters $G r^{*}$ and $\tilde{Z}_{\mathrm{L}}$ (provided the short chains are long enough to be entangled themselves). Hence, we plot them as solid lines in Fig. 6. However, we must recognize that the Viovy diagram is a two-dimensional projection of a three dimensional space defined by parameters $\left(\phi_{f}, Z_{\mathrm{L}}, Z_{\mathrm{S}}\right)$. For pure reptation, this projection works perfectly and all lines on the diagram are fixed. But when CLF is considered, some "lines" are no-longer perfectly projected in a twodimensional representation. The best that can be done is to choose a $2 \mathrm{D}$ projection which fixes some lines (as we have done above) and then discuss the other transitions, as we now do.

It is possible that CR is so slow that CLF in the thin tube, and the transition to terminal reptation in the thin tube, occurs before any substantial CR has occurred at all. This will be the case if $\tau_{\text {hop }}>\tau_{R}$, where $\tau_{\text {hop }} \approx 1.39 \tau_{\mathrm{CR}}$ sets the early-time crossover in Eq. (5). The result then is that the dynamics of reptation and CLF of the long chains is essentially unaffected by CR. Setting $\tau_{\mathrm{CR}}=\tau_{\mathrm{R}}$ gives the limit of this regime as

$$
G r^{*}=1.39 Z_{\mathrm{L}}^{-1} .
$$

Evidently the location of this transition on the Viovy diagram depends on the value of $Z_{\mathrm{L}}$, so it is not a fixed line in the projected $\left(\tilde{Z}_{\mathrm{L}}, G r^{*}\right)$ space. We represent it as a horizontal dashed line in Fig. 6, separating region $3 \mathrm{a}$ from $3 \mathrm{~b}$, and region $2 \mathrm{a}$ from $2 \mathrm{~b}$, choosing a representative value $Z_{\mathrm{L}}=100$, which is typical for many literature binary blends, giving $G r^{*}=0.0139$. Below this line, reptation and CLF of the long chains are unaffected by CR; above the line, some CLF in at least a partially diluted tube is possible.

Hence, below the horizontal line, we anticipate the terminal time of the long chains to be simply

$$
\tau_{\mathrm{dL}}=3 Z_{\mathrm{L}}^{3} f\left(Z_{\mathrm{L}}\right) \tau_{\mathrm{e}}
$$

On the other hand, above the line $G r^{*}=1.39 \tilde{Z}_{\mathrm{L}}^{-1}$, we anticipate CLF in the fat tube to accelerate the terminal relaxation. Provided $G r^{*}<0.047 \tilde{Z}_{\mathrm{L}}$ (i.e., chain motion along the thin tube dominates), the terminal time of the long chains will be modified to

$$
\tau_{\mathrm{dL}}=3 Z_{\mathrm{L}}^{3} f\left(\tilde{Z}_{\mathrm{L}}\right) \tau_{\mathrm{e}}
$$

where the function $f\left(\tilde{Z}_{\mathrm{L}}\right)$ accounts for the accelerating effects of CLF in the fatter tube. Intermediate between these two we expect CR to begin allowing CLF in a partially dilated tube. For a given effective dilution $\phi^{*}$, we can obtain both the time $\tau_{\mathrm{e} . f}^{*}=1.39 \tau_{\mathrm{CR}}\left(\phi^{*}\right)^{-2}$ required for CR-Rouse motion to locally relax the chain to the tube diameter corresponding to that dilution, and the CLF time $\tau_{\mathrm{R}} / \phi^{*}$ $=Z_{\mathrm{L}}^{2} \tau_{\mathrm{e}} / \phi^{*}$ for the chain in the tube at that dilution. Equating these two gives a partially diluted "supertube," in which the CLF time equals the time taken to explore the tube locally by $\mathrm{CR}$, and which is therefore the optimal diluted tube for CLF before transition to terminal reptation dynamics. This gives

$$
\phi^{*}=\frac{1.39 \tau_{\mathrm{CR}}}{Z_{\mathrm{L}}^{2} \tau_{\mathrm{e}}}=\frac{1.39}{Z_{\mathrm{L}} G r^{*}} .
$$

Equivalently, the number of effective "entanglements" in that supertube is $Z_{\mathrm{L}} \phi^{*}=1.39 / G r^{*}$. Hence we anticipate a terminal time

$$
\tau_{\mathrm{dL}}=3 Z_{\mathrm{L}}^{3} f\left(1.39 / G r^{*}\right) \tau_{\mathrm{e}},
$$

which, in regions $2 \mathrm{~b}$ and $3 \mathrm{~b}$, naturally interpolates between the limits in Eqs. (19) and (20).

We may now follow the arguments of Viovy et al. and compare the terminal reptation time of the long chains with other relaxation processes. If $\tau_{\mathrm{dL}}<\tau_{e, f}$, then the long chains reptate before they have fully explored the fat tube, and so entanglements between long chains do not determine the terminal modulus. It is as though the long chains are dilute and unentangled with other long chains. On the other hand, if $\tau_{\mathrm{dL}}$ $>\tau_{e f f}$, then the long chains locally explore the full fat tube before reptating, and so the terminal modulus is set by entanglements between long chains. Setting $\tau_{\mathrm{dL}}=\tau_{e, f}$ gives the boundary between these regimes. For $G r^{*}<1.39 Z_{\mathrm{L}}^{-1}$, we obtain

$$
G r^{*}=\frac{1.39}{3 \tilde{Z}_{\mathrm{L}}^{2} f\left(Z_{\mathrm{L}}\right)}
$$

a line with slope -2 on the Viovy diagram, whose position changes weakly with $Z_{\mathrm{L}}$, shown separating regions $3 \mathrm{a}$ and $2 \mathrm{a}$ in Fig. 6. For $G r^{*}>1.39 Z_{\mathrm{L}}^{-1}$, the transition $\tau_{\mathrm{dL}}=\tau_{e, f}$ must be solved numerically using Eq. (21) for $\tau_{\mathrm{dL}}$, giving a line with a slightly increasing gradient, as shown via the sloped dashed line separating regions $2 b$ and $3 b$ in Fig. 6.

Finally, for dilute long chains we may compare the reptation time $\tau_{\mathrm{dL}}$ with the CR Rouse time $\tau_{\mathrm{RCR}}$ of dilute long chains. This comparison indicates whether terminal relaxation by reptation or terminal relaxation by CR is the faster process. Using Eq. (21) for $\tau_{\mathrm{dL}}$, and the scaling result $\tau_{\mathrm{RCR}}=Z_{\mathrm{L}}^{2} \tau_{\mathrm{CR}}$, this transition occurs where

$$
3 G r^{*} f\left(1.39 / G r^{*}\right)=1 .
$$

However, the slip-spring model results, discussed in the Appendix B, indicate that the exact transition occurs at

$$
3 G r^{*} f\left(1.39 / G r^{*}\right)=0.047,
$$

where the factor 0.047 appears for the same reason as in Eq. (14), from an assessment of the exact scaling of friction from CR events. Numerical solution of Eq. (24) gives $G r^{*}$ $\approx 0.0254$, the solid horizontal line shown separating regions 1 and $2 \mathrm{~b}$ in Fig. 6. The main source of this small critical value of the Graessley parameter $\left(G r_{c}=0.0254\right)$ is that (as discussed in Subsection 2 of Appendix C) the slip-spring simulations exhibit a much faster rate of $\mathrm{CR}$ relaxation than 
would be expected from simple scaling arguments. The physical source of this rapid CR relaxation is likely that the tube takes finite sized hops for each CR event, rather than continuous Rouse motion.

\section{B. Checking the diagram with slip-spring simulations}

It is vital, now, to assess whether the modified diagram we have constructed is useful in mapping real data on binary blends. We may begin with an initial check: Are the slipspring simulations correctly located on the diagram? The horizontal co-ordinate on the diagram for the simulations is obtained by using the number of fat tube entanglements for the $N=512$ chains deduced above $(\tilde{Z}=12.5)$. The modified Graessley parameter can be obtained from Eq. (11), but noting that the slip-springs provide extra friction which slows down the reptation. Hence, we use $G r^{*}=k_{\mathrm{SL}} Z_{\mathrm{L}} \tau_{\mathrm{e}} / \tau_{\mathrm{CR}}$, where $k_{\mathrm{SL}}=\left(1+\zeta_{\mathrm{SL}} / \zeta\right) \approx 1.93$ represents the retardation of reptation due to slip-spring friction. For the chosen simulation parameters, $\tau_{\mathrm{e}}=N_{e}^{2} \zeta b^{2} / 3 \pi^{2} k_{\mathrm{B}} T=0.808$ and $Z_{\mathrm{L}}=105$. Hence, the simulations are located on the vertical blue dotted line, indicated on the left hand diagram of Fig. 6 . We may observe that the $\tau_{\mathrm{CR}}=300$ data lie on the boundary given by Eq. (14) where chain motion along the thin tube and CR transport along the fat tube are competing. This matches well with Fig. 3, where the plateau of the $\tau_{\mathrm{CR}}=300$ data lies just a little way above the horizontal dashed line (which is the expected limit of motion considering no CR transport along the fat tube, i.e., including only chain transport along the thin tube). For $\tau_{\mathrm{CR}}<300$, the CLF motion along the fat tube is clearly accelerated by CR; hence, these data lie in region 4 of the modified Viovy diagram. The data for $\tau_{\mathrm{CR}}=3000$, however, lie in region $3 \mathrm{~b}$ of the diagram, indicating that we expect some CLF within a dilating tube before transition to terminal reptation. This, again, can be seen in Fig. 3, where the enhanced CLF in a dilating tube is clearly visible, but no clear plateau is observed for CLF in the fat tube, before transition to terminal reptation. Finally, the data for $\tau_{\mathrm{CR}}=30000$ lie below the horizontal dashed line on the left of Fig. 6. Hence, we expect only a marginal effect of CR on the relaxation pathway. This, again, can be observed in Fig. 3 where the onset of $\mathrm{CR}$ for the $\tau_{\mathrm{CR}}$ $=30000$ data coincides with the transition to terminal reptation in the no CR data; hence the effect of CR is weak.

We can make one further check of our modified diagram against slip-spring simulation data. To the left of the diagram, we consider dilute long chains, which can be modeled by slip-spring simulations in which all slip-springs undergo CR. An example set of data of this type was shown in the right hand side of Fig. 5. The terminal relaxation for such blends will either be via CR Rouse (region 1 of the diagram) or reptation (region 2 of the diagram), with a transition between these regimes estimated at $G r^{*} \approx 0.0254$ above. For the simulations in Fig. $5(N=512, Z=105)$, this crossover corresponds to $\tau_{\mathrm{CR}} \approx 6500$, i.e., somewhere between the $\tau_{\mathrm{CR}}=3000$ and $\tau_{\mathrm{CR}}=30000$ simulations. Visual inspection of Fig. 5 indicates that certainly the terminal time of the $\tau_{\mathrm{CR}}=30000$ data is comparable to the data without $\mathrm{CR}$, which suggests that the terminal relaxation is driven by reptation. Likewise, the terminal relaxation for $\tau_{\mathrm{CR}}=3000$ and below is accelerated, though this could be either due to enhanced CLF or due to full relaxation via CR. We can test this by noting that the CR Rouse relaxation time $\tau_{\mathrm{RCR}}$ is proportional to $\zeta_{\mathrm{CR}}$, the effective friction constant per bead for CR Rouse motion. Hence, if we divide the time axis by $\zeta_{\mathrm{CR}}$, then the data for which terminal relaxation is via CR Rouse motion should achieve terminal relaxation at the same value of $t / \zeta_{\mathrm{CR}}$. We illustrate this data reduction in Fig. 7, using the full expression for $\zeta_{\mathrm{CR}}$ given in Appendix A. It is clear that the data for $\tau_{\mathrm{CR}} \leq 300$ relax at the same value of $t / \zeta_{\mathrm{CR}}$ (with peaks indicated by the vertical line) and so are relaxing by CR Rouse motion. The data for $\tau_{\mathrm{CR}}=3000$ relax slightly earlier, and so are close to the crossover. The data for $\tau_{\mathrm{CR}}=30000$ are very much accelerated in this representation, indicating that another relaxation mechanism (i.e., reptation) is responsible for the terminal relaxation.

A similar conclusion can be drawn from the simulations in [21], which were for shorter chains with $N=128$ and so $Z=26$. Simulations were performed in which all slip-springs underwent $\mathrm{CR}$ with a fixed average CR time just as with the data in Fig. 5. For these parameters, we therefore expect the crossover at $G r^{*} \approx 0.0254$ to correspond to a CR time of $\tau_{\mathrm{CR}}$ $\approx 1600$. Figure 11 (b) of [21] indicates that the crossover from terminal relaxation by $\mathrm{CR}$ to terminal relaxation by reptation occurs in the vicinity of $1000<\tau_{\mathrm{CR}}<2000$. It is clear from those data that the crossover is broad and smooth, and that close to the transition the terminal relaxation is faster than would be expected either from $\mathrm{CR}$ or from reptation acting alone.

\section{Checking the diagram with experimental binary blend data}

We may now proceed to locate data for literature experimental binary blends on our modified Viovy diagram, as shown on the right hand side of Fig. 6. The original sources

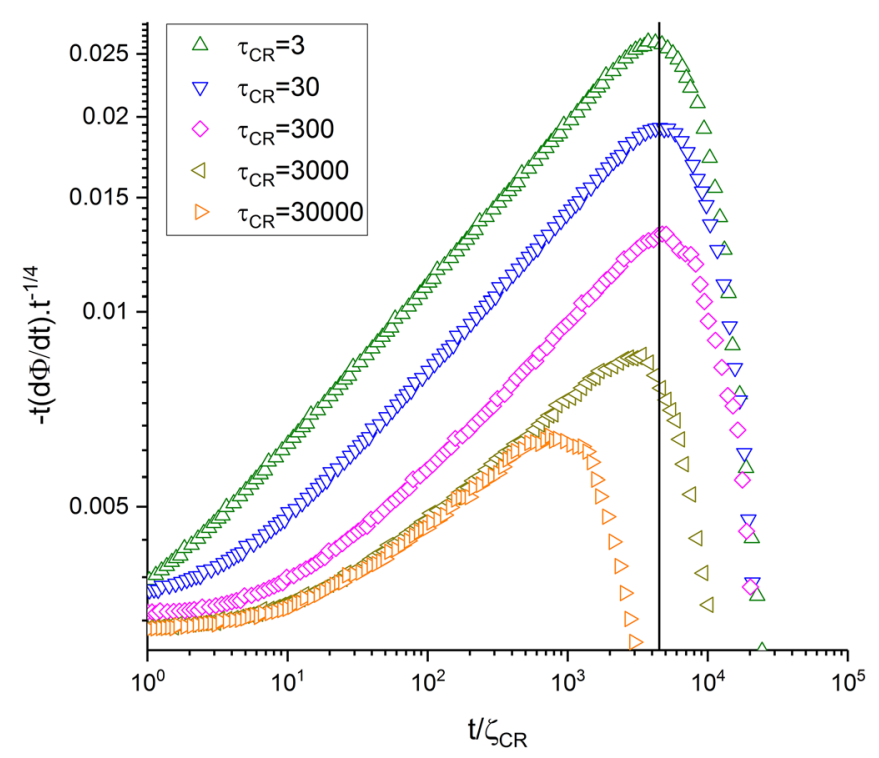

FIG. 7. Data from the right-hand side of Fig. 5 replotted against a horizontal axis of $t / \zeta_{\mathrm{CR}}$. The vertical line indicates the rough location of the peak for the data with $\tau_{\mathrm{CR}}=3,30$, and 300 . 
for the various data are listed in Table I. The majority of these data are collated in the publications of Park and Larson [14,15] and van Ruymbeke et al. [12]; the remaining data may be found in Read et al. [11], Watanabe et al. [16,17], Sawada et al. [13], and Struglinski and Graessley [19].

We focus first on the data in which the long chains are not dilute $(\tilde{Z}>1)$. Practically all such data in the literature fix the length of the long and short chains and then progressively dilute the long chains in the shorter ones. Hence, each set of such data corresponds to fixed values of $Z_{\mathrm{L}}$ and $Z_{\mathrm{S}}$, and so a fixed value of $G r^{*}$ [which can be calculated from Eq. (11)]. Thus, each set of data follows a horizontal line on the diagram. For each set, we calculate $G r^{*}$ and then represent the range of values of $\tilde{Z}$ available in the blend data with a line in Fig. 6. For this calculation, we require values for $M_{e}$, which we take to be $4.82 \mathrm{~kg} / \mathrm{mol}$ [polyisoprene (PI)], $1.81 \mathrm{~kg} / \mathrm{mol}$ [polybutadiene (PB)], and $16.5 \mathrm{~kg} / \mathrm{mol}$ [polystyrene (PS)]. These are the values given in the "Materials Database" of the REPTATE software ${ }^{1}$ and are based on fitting the LikhtmanMcLeish theory [9] for monodisperse linear polymers to literature data. Hence, these values are based on the definition $M_{e}=(4 / 5)\left(\rho R T / G_{N}^{0}\right)$, where $G_{N}^{0}$ is the plateau modulus [8]. However, the qualitative conclusions of this exercise are not strongly affected by the precise value for $M_{e}$ used within typical variation found in the literature, and the factor $\frac{4}{5}$ in its definition does not make a large difference to the positioning of blends on the logarithmic axes of the Viovy diagram.

For each set of data, we focus on a specific question: Is the terminal relaxation of the long chains observably accelerated upon dilution with short chains? For most data, this is visibly and unambiguously clear from the linear rheology. Nevertheless, it is instructive to note the work of Park and Larson [15] who examined whether or not their model, using reptation along the thin tube only, could describe several of these sets of data. Since their model did not include CLF along the fat tube we can assume that, if the data were adequately represented by their thin tube model, the terminal relaxation of long chains is not significantly accelerated by the short chains.

Further help is available in assessing the data presented by Watanabe et al. [16,17] for the PS 294/XX, PS 407/XX and PS 1070/XX blends. In those papers, the linear rheology data were converted to relaxation spectra, also presented in their papers, and these spectra were used to assess characteristic relaxation times, including the terminal relaxation of the long chains. For these data, then, the authors of the paper themselves indicate whether this terminal relaxation is accelerated.

In Fig. 6, green lines indicate blends where it is very clear that relaxation of long chains is accelerated by dilution with shorter chains. Blue lines indicate blends where there is no evidence that long chain relaxation is accelerated by shorter chains. The purple lines indicate two marginal cases where Park and Larson [15] found only a small acceleration of the long chains by the shorter ones as compared to predictions of their model with reptation along the thin tube, and one similar dataset from Watanabe et al. [16] which we discuss in detail below.

${ }^{1}$ REPTATE software available from http://reptate.com/ (2009 release) and http://reptate.readthedocs.io/en/latest/ (2018 release).
TABLE I. Literature references for data shown in Fig. 3.

\begin{tabular}{lcc}
\hline \hline Data & References & $\begin{array}{c}\text { Long chains accelerated } \\
\text { by short chains? }\end{array}$ \\
\hline PI 226/23 & Read et al. [11] & Yes \\
PI 308/21 & Watanabe et al. [31,32] & Yes \\
PI 308/94 & Watanabe et al. [31,32] & No \\
PI 329/14 & Sawada et al. [12,13] & Yes \\
PI 483/34 & Read et al. [11,28] & Yes \\
PI 626/nn & Sawada et al. [13] & Yes (dilute long chains) \\
PB 160/25 & Lee et al. [33] & Yes (marginal) \\
PB 181/39 & Struglinski and Graessley [19] & No \\
PB 335/71 & Rubinstein and Colby [34] & No \\
PB 410/3.9 & Wang et al. [35] & Yes \\
PB 410/110 & Wang et al. [35] & No \\
PB 450/39 & Struglinski and Graessley [19] & No \\
PB 450/93 & Struglinski and Graessley [19] & No \\
PB 550/20 & Park and Larson [14] & Yes \\
PS 294/36 & Watanabe et al. [16] & Yes \\
PS 294/83 & Watanabe et al. [16] & Yes \\
PS 294/161 & Watanabe et al. [16] & Yes (partial) \\
PS 407/22 & Watanabe et al. [17] & Yes \\
PS 407/36 & Watanabe et al. [17] & Yes \\
PS 1070/22 & Watanabe et al. [17] & Yes \\
PS 1070/36 & Watanabe et al. [17] & Yes \\
PS 1070/68 & Watanabe et al. [17] & Yes \\
PS 670/160 & Montfort al [36] & Yes (marginal) \\
\hline \hline & &
\end{tabular}

\section{Data in region 4 of the Viovy diagram}

The vast majority of the experimental data match very clearly the expectations of the modified Viovy diagram. In region 4 of the diagram, we anticipate that reptation and CLF in the fat tube, at a rate set by the CR from the shorter chains, will be faster than reptation and CLF in the thin tube. Hence, dilution with shorter chains will certainly accelerate the relaxation of the longer chains, both due to the accelerated reptation motion and from CLF in the fat tube. This is exactly what is observed in the data spanning this region. It is important therefore to include the CR contribution to CLF in the fat tube when describing data in this region, as was shown (for example) by van Ruymbeke et al. [12] in modeling the PI 308/21 data.

\section{Data in region $3 c$ of the Viovy diagram}

Acceleration of the long chains is also clearly observed in data found in region $3 \mathrm{c}$ of the diagram, notably in the PI 483/34 and PB 550/20 data. Some of the PS 294/83 data also lie within this region. In this region, chain motion along the thin tube is expected to be faster than motion by $\mathrm{CR}$ along the fat tube: Hence, the original work of Viovy et al. predicted no acceleration of the terminal time. However, we now predict that CR gives the long chains freedom for CLF in the fat tube, and that this provides a mechanism to accelerate the terminal relaxation. This explains the speeding up of the long chains for these data, as was shown explicitly for the PI 483/34 data by Read et al. [11] by fitting the data with their model.

For the PI 483/34 and PB 550/20 data, we may test to see whether the expected speeding up of the terminal relaxation 
TABLE II. Dilution factors obtained from self-consistent formula $\phi$ $=\phi_{\text {chem }} \sqrt{f\left(\phi Z_{L}\right)}$ for PI438/34 and PB550/20.

\begin{tabular}{|c|c|c|c|}
\hline \multicolumn{2}{|c|}{ PI438/33 } & \multicolumn{2}{|c|}{ PB550/20 } \\
\hline$\phi_{\text {chem }}$ & $\phi$ & $\phi_{\text {chem }}$ & $\phi$ \\
\hline 1.0 & 0.822 & 1.0 & 0.900 \\
\hline 0.4 & 0.283 & 0.2 & 0.154 \\
\hline 0.2 & 0.114 & 0.1 & 0.066 \\
\hline 0.1 & 0.0398 & 0.05 & 0.025 \\
\hline
\end{tabular}

of long chains is well predicted by Eq. (20) (i.e., including only the accelerating effect of CLF in the fat tube). In order to do this, we must decide on an appropriate dilution factor $\phi$ to apply in calculating $\tilde{Z}_{\mathrm{L}}=\phi \mathrm{Z}_{\mathrm{L}}$. At first sight, it may seem appropriate to use $\phi=\phi_{\text {chem }}$, where $\phi_{\text {chem }}$ is the chemical volume fraction of the long chains in the melt. However, as was pointed out previously [11], this underestimates the degree of dilution appropriate for the fat tube, since a significant portion of the long chains relax on the same fast timescale as the short chains (e.g., by CLF). In [11], the fraction $\phi$ was treated as a fitting parameter. Here we construct an approximate argument to estimate the maximal dilution that could reasonably be applied (consistent with a dilution exponent of 1). We suppose that during CLF in the fat tube, all long chain material relaxing due to CLF acts to give CR on the remaining, unrelaxed long chain material, further widening the fat tube. Hence, the dilution factor that should be applied at the timescale of CLF in the fat tube is $\phi=x_{L} \phi_{c h e m}$, where $x_{L}$ is the fraction of long-chains that remain unrelaxed by CLF. Further, we can estimate $x_{L}$ by noting that the main contribution to speeding up reptation by CLF is that it shortens the distance required for the chains to diffuse. Since the diffusive relaxation time scales as the square of the diffusion distance, we expect $x_{L} \approx \sqrt{f\left(\tilde{Z}_{\mathrm{L}}\right)}=\sqrt{f\left(\phi Z_{L}\right)}$ (at least for well entangled chains). This gives a self-consistent formula $\phi=\phi_{\text {chem }} \sqrt{f\left(\phi Z_{L}\right)}$ which can be solved numerically to obtain $\phi$. We perform this exercise for the PI 483/34 and PB $550 / 20$ blends, resulting in the dilution factors listed in Table II. These may then in turn be used to estimate the terminal relaxation times using Eq. (20).

We can, additionally, estimate the degree to which CR motion, carrying the chain along the fat tube, further accelerates the terminal relaxation of the long chains. Although these blends are in region $3 \mathrm{c}$ of the modified Viovy diagram, so that motion along the thin tube is dominant, alongtube $\mathrm{CR}$ motion can still provide a perturbation to the chain relaxation. We estimate the result of this by including the full expression [Eq. (2)] for the effective friction for motion along the fat tube, in which the second term gives the accelerating effect due to CR. This is done in Subsection 2 of Appendix C, giving rise to a new Eq. (C2) for the long chain terminal relaxation time. The correction factor $A_{C R}$ in this expression can become as small as 0.6 for the most dilute blend considered here in the PI483/34 series, indicating a significant extra correction (though the dominant correction remains the acceleration due to CLF in the fat tube).
We show linear rheology data for the two blend series in Figs. 8(a) and 8(c) and indicate the predicted terminal times from Eq. (20) (only acceleration due to CLF in fat tube) using dashed vertical lines and using Eq. (C2) (additional acceleration due to $\mathrm{CR}$ along fat tube) using solid vertical lines. From Figs. 8(a) and 8(c), we may already make the following comments. Equation (20) predicts a substantial acceleration of the terminal relaxation of the long chains, simply due to the accelerating effects of CLF. This predicted acceleration is commensurate with the acceleration observed in the data, especially since we have noted that the effective dilution should be stronger than the long chain fraction as estimated above. In addition, CR motion along the fat tube provides a further accelerating effect [which we include using Eq. (C2)]. This becomes more significant as dilution increases and the boundary with region 4 of the Viovy diagram is approached.

However, we note that, for these data, the cross-over frequency (where $G^{\prime}=G^{\prime \prime}$ ) is not a good indicator of the terminal time, since the relaxation spectrum changes appreciably upon dilution. In order to check more quantitatively whether the predicted acceleration is accurately modeled, we require some estimate of the longest relaxation time present in the data. This is difficult to obtain, but one method that seems reasonably robust is to fit the data with a spectrum of Maxwell modes using the software REPTATE. The fitting procedure in this software uses a set of modes with evenly spaced relaxation times on a logarithmic scale, where the relaxation time of the fastest and slowest modes are themselves free fitting parameters. Hence, the relaxation time of the slowest fitted mode with a substantial modulus is a reasonable estimate of the longest substantial relaxation time in the spectrum. It can be checked that the result is robust, by starting the minimization procedure from different initial conditions and with different numbers of modes. We show, in Figs. 8(b) and 8(d), plots of the longest relaxation times for the data as a function of dilution, along with the predictions from Eq. (20) as a dashed line and Eq. (C2) as a solid line. The predictions for the PI 483/34 data are excellent, indicating that CLF in the fat tube gives the dominant accelerating effect, with corrections due to $\mathrm{CR}$ along the fat tube. At the lowest long chain fractions, our approximate procedure for estimating the appropriate $\phi$ (Table II) may overestimate the dilution due to CLF, since the resulting acceleration is too strong. For the PB 550/20 data, the predictions are also good, but with one caveat: The fitting procedure to estimate the longest relaxation time of the pure PB550 melt seems to give a surprisingly long relaxation time, suggesting an additional (roughly $30 \%$ ) speeding up of the relaxation of the PB550 chains upon initial dilution with PB20, which is not captured by Eq. (20) or (C2). If we instead use the cross-over frequency (where $G^{\prime}=G^{\prime \prime}$ ) to estimate the relaxation time of the pure PB550 [shown by a solid star in Fig. 8(d)], then the predictions are much better, of a similar quality to the PI $438 / 34$ data. It may be that there is an artifact in the data for pure PB550, or that there is an additional accelerating effect on dilution with the PB20 chains for this particular series of materials which is not captured by the theory. 


\section{Data in region 3 and $2 b$ of the Viovy diagram}

Returning to the modified Viovy diagram of Fig. 6, we now consider regions 3 and $2 b$ of the diagram. In these regions, it is anticipated that some CR occurs, while CLFs are taking place, but that within the CLF timescale, there is not sufficient time for the chain to locally explore the full width of the fat tube by CR motion. So, the CR that does occur will permit an increased degree of CLF and this will partially accelerate the terminal relaxation of the long chains as compared to a melt of pure long chains. Here, the situation is nicely illustrated by our $\tau_{\mathrm{CR}}=3000$ data in Fig. 3: CR permits some enhanced CLF in a gradually widening tube, but before full exploration of the fat tube, there is transition to terminal reptation, which is slightly accelerated since the enhanced CLF reduces the distance required to reptate.

However, further dilution by the short chains will not further accelerate the long chain relaxation, because dilution only increases the diameter of the fat tube-but, as already stated, the chain cannot explore the full fat tube in the CLF timescale. Hence, further dilution does not lead to an increased amount of CLF, so there is no mechanism to additionally accelerate the long chain relaxation. Dilution will, however, reduce the modulus associated with the terminal

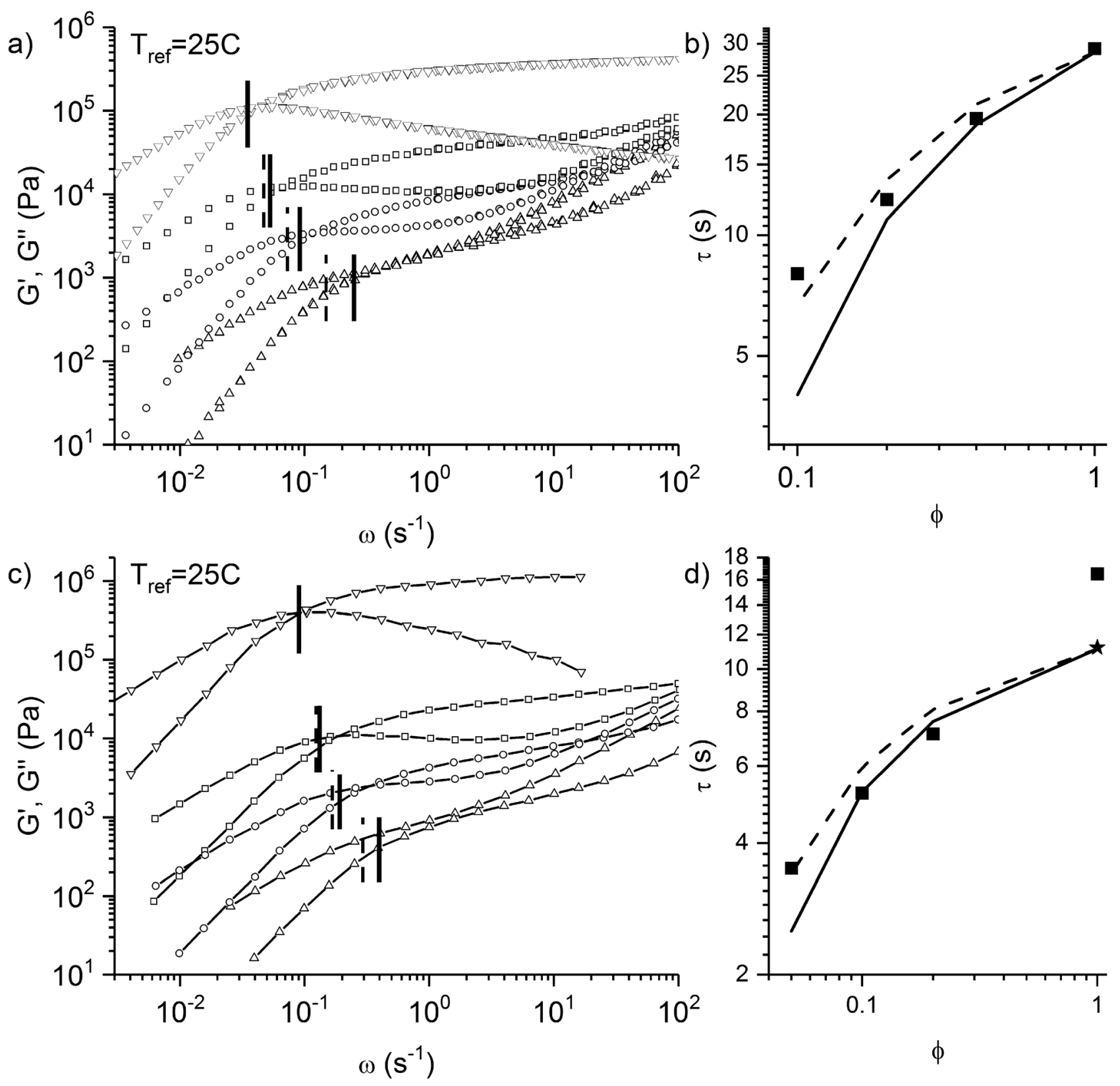

FIG. 8. (a) Linear rheology data for the PI $483 / 34$ data [11] at long chain fractions of $1,0.4,0.2$, and 0.1 . Dashed vertical lines are estimations of the inverse terminal time of long chains using Eq. (20), and solid lines using Eq. (C2) using $\tau_{\mathrm{e}}=1.4 \times 10^{-5} \mathrm{~s}, Z_{\mathrm{L}}=100$ and $\phi$ as given in Table II. (b) Longest relaxation times for the PI 483/34 data [11] as estimated by Maxwell fitting from REPTATE software (solid squares) together with predictions of long chain relaxation time using Eq. (20) (dashed line) and Eq. (C2) (solid line). (c) Linear rheology data for the PB 550/20 data [14] at long chain fractions of 1, 0.2, 0.1, and 0.05 . Dashed vertical lines are estimations of the inverse terminal time of long chains using Eq. (20), and solid lines using Eq. $(\mathrm{C} 2) \mathrm{using} \tau_{\mathrm{e}}=1.6 \times 10^{-7} \mathrm{~s}, Z_{\mathrm{L}}$ $=304$, and $\phi$ as given in Table II. (d) Longest relaxation times for the PB 550/20 data [14] as estimated by Maxwell fitting from REPTATE software (solid squares). Relaxation time as estimated from crossover frequency of pure PB550 data shown as a solid star. Predictions of long chain relaxation time using Eq. (20) (dashed line) and Eq. (C2) (solid line). 
relaxation. The modulus is expected to scale as $\phi^{2}$ in region $3 \mathrm{~b}$ (by the time of the terminal reptation time, the chain has explored the fat tube diameter) and as $\phi$ in region $2 \mathrm{~b}$ (effectively the long chains appear as dilute throughout their whole relaxation pathway).

A particularly interesting set of data in this region is the PS294/161 data of Watanabe et al. [16]. As noted above, for these data the authors determined the relaxation spectrum from the linear rheology data and used this to determine the terminal relaxation time (and terminal modulus) of the long chains. According to their presented results (Fig. 9 of [16]), there is a slight acceleration of the long chain relaxation time from the pure melt to $40 \%$ concentration of the long chains, but no further acceleration upon further dilution. The particular observation of no further acceleration upon dilution is exactly in keeping with the predictions described above. For most of the concentration range, the terminal modulus for these data scales roughly as $\phi$, though it may be that a stronger scaling is attained at the largest long chain fractions (Fig. 10 of [16]).

The PS 670/160 and PB 160/25 literature data are also found in region $3 \mathrm{~b}$ of the Viovy diagram. As noted above, for these data, Park and Larson [15] found a small acceleration of the long chains by the shorter ones as compared to predictions of their model with reptation along the thin tube.

\section{Data toward the lower part of the Viovy diagram}

Toward the lower part of the diagram (e.g., region 3a) CR from the short chains is too slow to produce any appreciable acceleration of the long chains. This prediction, again, is borne out by the data.

Only one set of data appears inconsistent with the diagram as drawn, the PB 450/39 data of Struglinski and Graessley [19]. As indicated in the diagram, this data set is to the extreme lower end of region $3 \mathrm{c}$. We therefore expect that CLF in the fat tube might accelerate the long chains when diluted in the shorter chains. The situation is marginal, however: The data are extremely close to the 3 a region near the bottom of the diagram. This, combined with the relatively narrow range of dilutions present in the data, may be the reason why acceleration of long chains is not obviously present. It would be valuable to generate further data in the $3 \mathrm{c}$ region of the diagram.

\section{Data for dilute long chains}

We may also compare the modified diagram with blend data obtained in the limit of dilute long chains. Sawada et al. [13] present several sets of data in which the terminal time of dilute, long PI chains is monitored as a function of length of the short chain matrix polymers. In particular, they examine the extent to which the terminal time follows the expected scaling for CR Rouse (CRR) relaxation of long chains. They find that, as the length of short chains is increased, the CRR scaling is followed until, above a particular molecular weight of short chains, the relaxation is faster than expected from pure CR motion and there is then a broad transition toward the reptation time of pure long chains. The CCR scaling in their data is only approximate since the CR relaxation time of the long chains scales as $\tau_{\mathrm{RCR}} \sim Z_{L}^{2} Z_{S}^{\gamma}$ where the exponent $\gamma$ appears to take a value of 3 , or a little less, rather than the exponent of 3.4 which might be expected from the variation of short chain relaxation time with molecular weight. As discussed in Subsection 2 of Appendix C, the likely cause of this is a surprisingly broad transition to the limit of fast CR, where the bare Rouse friction of long chains slows the rate of long chain relaxation. We predict that this transition zone extends up to short chain lengths of around 10 entanglements, resulting in apparent scaling exponents $\gamma$ which could be anywhere between 1 and 3 depending on the length of the short chains.

The clearest set of data from Sawada et al. [13] is for PI of molecular weight $626 \mathrm{~kg} / \mathrm{mol}$, diluted in shorter chains. These data appear to show the approximate CRR scaling for matrix short chain molecular weights up to roughly $60 \mathrm{~kg} /$ mol, corresponding to a modified Graessley number of $G r^{*}$ $\approx 0.066$. We represent these data with the red vertical line on Fig. 6. The remainder of their data, for larger matrix molecular weights, span the broad transition region toward the reptation time of pure long chains; we represent these data by the brown vertical line in the right hand side of Fig. 6. We can compare these data with the critical $G r^{*} \approx 0.0254$ predicted on the basis of slip-link simulation data above. The data should be expected to deviate from the CRR scaling as this critical $G r^{*}$ is approached, which is exactly the observation of Sawada et al. Data at other long chain molecular weights show a similar behavior, e.g., for long chain molecular weight $329 \mathrm{~kg} / \mathrm{mol}$, the transition appears to begin at a short chain molecular weight of $43 \mathrm{~kg} / \mathrm{mol}\left(G r^{*} \approx 0.113\right)$. The work presented here may be the first explanation of the low critical value of the Graessley parameter $G r^{*} \ll 1$ for dilute long chains. As noted above, the main reason for this is that relaxation due to CR is faster than would be anticipated from simple scaling arguments, as discussed in Subsection 1 of Appendix C.

\section{Summary}

We may summarize this section as follows. We have redrawn the Viovy diagram in the light of theoretical insight regarding the effects of CLF on the relaxation of long chains in binary blends, in particular, noting that CLF in the fat tube, or in a widening tube, is possible even when the chain motion is primarily along the thin tube. This insight is confirmed by the slip-spring simulations we performed. The simulations also permit us to quantitatively specify the positions of lines on the diagram, rather than simply make scaling arguments (e.g., for dilute long chains we predict a critical Graessley number $G r^{*} \approx 0.0254 \ll 1$ ). By locating experimental and simulation data on the diagram, we were able to confirm the qualitative and quantitative predictions of the redrawn diagram.

\section{CONCLUSION}

The goal of this paper has been to use idealized simulations, based on Likhtman's slip-spring model [3], to inform our understanding of the interaction between CR and CLFs in 
entangled linear polymer chains (and particularly binary blends). We began with a set of simulations in which the CR rate was precisely defined for a fraction of the slip-springs (representing CR from short chains in a binary blend system), while CR was suppressed for the remaining slip-springs (representing slow CR from entanglements with long chains). Our data analysis revealed regimes corresponding to CLF in the "thin tube" (at early times), CLF in a fat tube (at late times) and an intermediate regime in which CLF occurs in an effectively dilating tube. The effective friction for motion along the fat tube was found to be well described by the framework of Read et al. [11,21]. Furthermore, we derived a simple theoretical model based on scaling arguments for Rouse-chain motion, which was able to describe the full time dependence of the simulation data in the $\mathrm{CR}$ regime for slower CR rates.

In this context, we may briefly discuss the apparent success of applying a tube model description for the results of a slip-spring model, both in this paper and in our previous publication [21]. The success of this mapping between slipspring and tube models is a significant result of this work. As noted in the Introduction, for chain motion in a fixed tube (or with fixed slip-springs), we may reasonably expect that the chain motion (reptation and CLF) would be similar in both models; this corresponds to "motion along the thin tube" in the language of the tube model. What is less obvious is that CR will give exactly analogous motion in the two models. One particular question that presses is why Eq. (2) should apply, specifically why and how does CR motion give an accelerated motion as though "along the fat tube" in the slipspring model? We may note that the fat tube in the slipspring model is defined by the slow slip-springs which do not undergo CR. The rate of chain motion through these slow slip-springs (i.e., along the fat tube) is governed by the motion of the chain sections trapped between two consecutive slip-springs. It is only appropriate to speak about the fat tube on timescales such that the chain between two consecutive slow slip-springs can locally equilibrate. In this case, the rate of chain motion through slow slip-springs is governed by the equilibration time of such chain sections, which is directly determined by the CR rate of the faster slip-springs. Consideration of this gives rise to an equation of the form of Eq. (2), even though this equation was originally conceived based on tube model ideas.

Having adequately understood the simulation data, we then sought to draw out the implications for experimental studies of entangled binary blends of linear polymers, and especially for the dynamics of the long chains in such liquids. We revisited the diagram produced by Viovy et al. [18], relocating some of the lines on the diagram and identifying new regimes, based on the physics and quantitative information implied by the simulation data. In particular, we identified the new region in the diagram in which along-tube motion of the long chains is predominantly along the contour of the thin tube, yet CLF is possible to the extent allowed by the fat tube, resulting in an acceleration of the terminal relaxation. We also provide what may be the first explanation of the surprisingly low critical Graessley number for dilute long chains. We were able, successfully, to locate a wide range of literature data on our redrawn diagram.
We anticipate that this present study will prove useful in several contexts. Although the work of Viovy et al. has been known for some time, we believe this is the first time a large amount of literature data has been quantitatively and successfully located on such a diagram. We hope, therefore, that this diagram will assist further studies on binary blends, in particular providing a map on which to locate experimental systems and guiding the choice of suitable blends for future study. We are also certain that this work will assist studies that go beyond simple binary blends, toward the study of fully polydisperse systems which are (obviously) the types of liquids more commonly encountered in an industrial context. In particular, the scaling model developed in Sec. III B can be generalized to more complicated environments with multiple CR times.

\section{ACKNOWLEDGMENTS}

The authors are grateful to Jay Schieber for providing us access to computational resources of the Center for Molecular Study of Condensed Soft Matter of Illinois Institute of Technology ( $\mu \mathrm{CoSM} / \mathrm{IIT})$. The authors acknowledge helpful discussions with Chinmay Das, Tom McLeish, Victor Boudara, Hiroshi Watanabe, and Evelyne van Ruymbeke. They are also grateful for the useful questions asked by anonymous reviewers. This paper is dedicated to the memory of our friend, and coauthor, Professor Alexei Likhtman.

\section{APPENDIX A: MAPPING FROM THE SLIP-SPRING MODEL TO TUBE MODEL}

This paper uses the slip-spring model [3] for the simulations presented. As indicated in the text, these simulations are closely approximated by tube model calculations, but the mapping from slip-spring model to tube model requires accounting for (i) the fact that slip-springs are not pointlike constraints, and (ii) slip-springs give a non-negligible contribution to the effective friction for chain sliding along the tube axis. These factors were discussed in detail in an earlier publication [21] and we here summarize the main results of that work and their application in this paper. For more detailed explanations, see [21].

The fact that slip-springs permit the chain to fluctuate about the slip-spring point of attachment allows the chain greater freedom than would be the case if the constraints were pointlike. Hence, the effective number of beads per entanglement in the tube model, $N_{e}$, is greater than the number of beads per slip-spring, $N_{e, s s}$. We obtained an approximate relationship between them as

$$
N_{e}=N_{e, s s} \sqrt{1+4 n_{s}},
$$

where $n_{s}=N_{s} / N_{e, s s}$. Correspondingly, as slip-springs are removed, the ratio of $N_{e} / N_{e, s s}$ changes, so that dilution of slipsprings is not identical to dilution of entanglements in the tube model. If slip-springs are diluted by factor $f$ (e.g., by CR), then the corresponding dilution factor in the tube model is

$$
\phi_{f}=f \frac{\sqrt{1+4 n_{s}}}{\sqrt{1+4 f n_{s}}} .
$$


These two equations were used in Sec. II to estimate $N_{e}$ and dilution factor $\phi_{f}$ for the fat tube.

Accounting for the above dilution effects, and for the effective slip-link friction per bead $\zeta_{\mathrm{SL}}$ for along tube motion, we found that the effective friction per bead for motion along the fat tube contour is

$$
\zeta_{\text {fat }}=\frac{f}{\phi_{f}} \zeta_{\mathrm{SL}}+\tilde{\zeta}_{\mathrm{fat}},
$$

where the first term gives the contribution from the slow slip-springs which define the fat tube, and the combined friction from chain sliding along the thin tube and from CR is obtained from

$$
\frac{1}{\tilde{\zeta}_{\text {fat }}}=\frac{\phi_{f}}{\zeta+(1-f) \zeta_{\mathrm{SL}}}+\frac{1}{(1-f) \zeta_{\mathrm{CR}}+\zeta^{+}},
$$

where

$$
\zeta^{+}=\frac{\zeta\left(\zeta+(1-f) \zeta_{\mathrm{SL}}\right)}{\left(1-\phi_{f}\right) \zeta+(1-f) \zeta_{\mathrm{SL}}}
$$

Equation (A4) is the slip-spring equivalent of Eq. (2), derived for the tube model.

The effective friction from CR hopping of the thin tube was found to be

$$
\zeta_{\mathrm{CR}}=\frac{2 \tau_{\mathrm{CR}} k_{\mathrm{B}} T}{b^{2} N_{e}^{2} \alpha_{\mathrm{CR}}^{2}}\left(1+\frac{1}{K} \sqrt{\frac{2 \zeta b^{2} N_{e}^{2} \alpha_{\mathrm{CR}}^{2}}{\tau_{\mathrm{CR}} k_{\mathrm{B}} T}}\right) .
$$

The second term in parentheses on the right of this equation is a correction accounting for the influence of chain friction on the hop length from CR events. Specifically, if CR is too fast, then the chain is not able to move a significant distance during a CR event, and so the hop length is decreased. By fitting Eq. (A6) to chain diffusion data in the slip-spring model [21], the otherwise unknown constants were found to be $\alpha_{\mathrm{CR}}=1.2$ and $K=0.36$.

Using the above equations in the scaling analysis presented in Sec. III A, we find that the plateau values for CLF in the fat tube to occur at

$$
-t^{\frac{3}{4}} \frac{\mathrm{d} \Phi}{\mathrm{dt}}=\frac{C_{\mu}}{4 Z}\left(\frac{3 \pi^{2} k_{\mathrm{B}} T}{\phi_{f}^{2} \zeta_{\mathrm{fat}} b^{2} N_{e}^{2}}\right)^{\frac{1}{4}}
$$

where $\zeta_{\text {fat }}$ is as given in Eq. (A3). This is the equation used to determine the level of the horizontal lines in Fig. 3.

The expected longest timescale for CLF in the fat tube (after which there is a transition to terminal reptation) can be obtained as the chain Rouse time subject to the fat tube friction, i.e.,

$$
\tau_{\mathrm{CLF}}=\frac{N^{2} \zeta_{\mathrm{fat}} b^{2}}{3 \pi^{2} k_{\mathrm{B}} T} .
$$

Finally, Eq. (A3) can be used directly in Eq. (3) for the integration to obtain the full time dependent curves in Fig. 5. In order to do this, $\phi_{f}$ in the above expressions must be replaced by $\phi$ from Eq. (5), and (correspondingly) $f$ is obtained by inverting the relationship A2 to obtain $f$ in terms of $\phi$.

\section{APPENDIX B: LINES ON THE REVISED VIOVY DIAGRAM FROM SLIP-SPRING PARAMETERS}

The quantitative description of results of slip-spring simulations presented in Appendix A and in the main body of the paper permits us to locate more accurately the lines on the Viovy diagram, as discussed in Sec. IV A. Here we specify the details of how these lines are obtained.

In order to determine whether chain motion along the thin tube, or CR motion along the fat tube, is the dominant process for chain transport (in reptation and CLF), we may compare the two terms in Eq. (2). The slip-spring equivalent of this is Eq. (A4). To good approximation (ignoring the contributions of slip-link friction, and assuming CR is slow) the crossover lies where

$$
\frac{\phi_{f}}{\zeta}=\frac{1}{\zeta_{\mathrm{CR}}} \approx \frac{b^{2} N_{e}^{2} \alpha_{\mathrm{CR}}^{2}}{2 \tau_{\mathrm{CR}} k_{\mathrm{B}} T}
$$

where we have used only the first term in Eq. (A6). This rearranges to give

$$
\phi_{f} \approx \frac{3 \pi^{2} \alpha_{\mathrm{CR}}^{2}}{2} \frac{\tau_{\mathrm{e}}}{\tau_{\mathrm{CR}}} .
$$

Finally, multiplying through by $Z_{\mathrm{L}}$ gives

$$
\tilde{Z}_{\mathrm{L}} \approx \frac{3 \pi^{2} \alpha_{\mathrm{CR}}^{2}}{2} G r^{*}
$$

which results in Eq. (14) since $\beta=2 /\left(3 \pi^{2} \alpha_{\mathrm{CR}}^{2}\right) \approx 0.047$.

For dilute long chains, a critical relaxation time is the $\mathrm{CR}$ Rouse time of the whole chain, which is the Rouse time subject to the CR friction $\zeta_{\mathrm{CR}}$

$$
\tau_{\mathrm{RCR}}=\frac{N^{2} \zeta_{\mathrm{CR}} b^{2}}{3 \pi^{2} k_{\mathrm{B}} T} .
$$

In the limit of slow $\mathrm{CR}$, this gives

$$
\tau_{\mathrm{RCR}}=\frac{2}{3 \pi^{2} \alpha_{\mathrm{CR}}^{2}} \frac{N^{2}}{N_{e}^{2}} \tau_{\mathrm{CR}} \approx 0.047 Z_{L}^{2} \tau_{\mathrm{CR}} .
$$

This, then, gives rise to Eq. (24).

Other lines on the diagram depend on the time $t=\tau_{e, f}$ for the thin tube to explore the width of the fat tube. This may be obtained from the late-time crossover in Eq. (5), which occurs when

$$
\frac{\tau_{\mathrm{hop}}}{\tau_{\mathrm{e}, \mathrm{f}}}=\phi_{f}^{2}
$$

Using $\tau_{\text {hop }}=c_{\phi}\left(N_{e}^{2} b^{2} \zeta_{\mathrm{CR}} / k_{\mathrm{B}} T\right) \approx 2 c_{\phi} \tau_{\mathrm{CR}} / \alpha_{\mathrm{CR}}^{2}$, we find that

$$
\tau_{\mathrm{e}, \mathrm{f}}=\frac{2 c_{\phi}}{\alpha_{\mathrm{CR}}^{2}} \tau_{\mathrm{CR}} \phi_{f}^{-2},
$$


which gives Eq. (16), since we use $c_{\phi}=1$ and $\alpha_{\mathrm{CR}}=1.2$, so $\frac{2 c_{\phi}}{\alpha_{\mathrm{CR}}^{2}} \approx 1.39$.

\section{APPENDIX C: DETAILED CORRECTIONS TO RELAXATION TIMES FOR REAL CHAINS}

This Appendix includes some details of estimating of relaxation times for binary blends of real polymers, specifically examining how the $\mathrm{CR}$ rate varies with short chain length, and corrections to the terminal reptation time of the long chains due to $\mathrm{CR}$ motion along the fat tube. The expressions used in this section can be found also in Appendix G of [21].

\section{Variation of long chain CR time with short chain length}

Using Eq. (A6) for the effective friction from CR hops, adding in the chain friction, and rewriting the resultant expression in terms of tube model units, the relaxation time of dilute long chains by CR was found to be $\{\mathrm{Eq}$. (G5) of [21]\}

$$
\tau_{\mathrm{RCR}}=Z_{L}^{2}\left[\beta \tau_{\mathrm{CR}}\left(1+\frac{1}{K} \sqrt{\frac{\tau_{\mathrm{e}}}{\beta \tau_{\mathrm{CR}}}}\right)+\tau_{\mathrm{e}}\right],
$$

where the parameter $\beta=\left(2 / 3 \pi^{2} \alpha_{\mathrm{CR}}^{2}\right) \approx 0.047$ and we use $\tau_{\mathrm{CR}}=\tau_{\mathrm{dS}}$. The above equation is equivalent to B5, but including the crossover to faster CR.

We may note two main differences between this expression and the more usual "scaling" expression $\tau_{\mathrm{RCR}}=Z_{L}^{2} \tau_{\mathrm{CR}}$ often found in the literature (see, e.g., [12,18,37]). First, we note that the factor $\beta \approx 0.047$ is small, which indicates that relaxation from $\mathrm{CR}$ is much faster than would be expected from the simple scaling argument. One result of this is that fits to data using the standard scaling $\tau_{\mathrm{RCR}}=Z_{L}^{2} \tau_{\mathrm{CR}}$ typically require surprisingly small values of $\tau_{\mathrm{CR}} \ll \tau_{\mathrm{dS}}[12,37]$.

A second issue is the broad crossover toward fast $\mathrm{CR}$ implied by the square root term in Eqs. (A6) and (C1). This term was obtained [21] by including the effect of the local Rouse friction of the long chain on the distance of the CR hop (for fast CR the chain does not have time to explore a significant distance in the CR time, so the hop distance is reduced). This expression gave a good match to the crossover observed in slip-spring simulations as the CR time was varied. We may illustrate the effect of this by plotting the ratio $\tau_{\mathrm{RCR}} / Z_{L}^{2}$ as a function of $Z_{S}$, under the assumption that $\tau_{\mathrm{dS}}=3 \tau_{\mathrm{e}} Z_{S}^{3} f\left(Z_{S}\right)$. We do this in Fig. 9, indicating both the result of the full Eq. (C1) and the leading order approximation $\tau_{\mathrm{RCR}}=Z_{L}^{2} \beta \tau_{\mathrm{dS}}$ for slow $\mathrm{CR}$. We note from this that the crossover region is, in fact, extremely and surprisingly broad, easily extending as far as $Z_{S}=10$ entanglements or more. This is a result of a combination of the square root term in Eq. (C1), and the small value of $\beta$.

A consequence of this, for realistic short chain lengths in the transition zone, is that the variation of long chain CR relaxation time with short chain length is typically weaker than the 3.4 power law that might be anticipated from a relation of form $\tau_{\mathrm{RCR}}=Z_{L}^{2} \tau_{\mathrm{dS}}$. Sawada et al. [13] found scalings $\tau_{\mathrm{RCR}} \sim Z_{S}^{\gamma}$ with $\gamma \approx 3$ or slightly less. Examining $\mathrm{CR}$ relaxation of stars, Ebrahimi et al. [37] proposed a relation of form $\tau_{\mathrm{CR}} \sim \tau_{\mathrm{dS}} / Z_{S}^{2}$ for the $\mathrm{CR}$ time, which gives $\tau_{\mathrm{RCR}}$ $\sim Z_{S}^{\gamma}$ with $\gamma$ in the range of 1 to 1.4. The dash-dot lines in Fig. 9 indicate that apparent scaling laws with $\gamma$ in the range of 1 to 3 are certainly possible to obtain, especially when the short chains have only a few entanglements.

\section{Correction to long chain reptation time from $\mathrm{CR}$ along the fat tube}

For blends substantially in region $3 \mathrm{c}$ of the modified Viovy diagram, we predicted that the reptation time of the long chains could be well approximated by Eq. (20), i.e., the bare reptation time corrected for CLF in the fat tube. On crossing into region 4 of the Viovy diagram, this terminal time is expected to be further accelerated by CR motion of the thin tube along the fat tube. In practice, however, lines of the diagram are not sudden transitions, and there is instead a broad transition zone from one region to the next, resulting in corrections to the reptation time as the lines on the Viovy diagram are approached. We may express the speeding up of reptation by CR events as a corrected version of Eq. (20)

$$
\tau_{\mathrm{dL}}=3 A_{C R} Z_{\mathrm{L}}^{3} f\left(\tilde{Z}_{\mathrm{L}}\right) \tau_{\mathrm{e}}
$$

where the CR correction factor was obtained in Appendix G of [21] as

$$
\frac{1}{A_{C R}}=1+\frac{(1-\phi)}{\phi\left[(1-\phi)^{2} \zeta_{\mathrm{CR}} / \zeta+1\right]},
$$

and

$$
\frac{\zeta_{\mathrm{CR}}}{\zeta}=\frac{\beta \tau_{\mathrm{dS}}}{\tau_{\mathrm{e}}}\left(1+\frac{1}{K} \sqrt{\frac{\tau_{\mathrm{e}}}{\beta \tau_{\mathrm{dS}}}}\right) .
$$

Equations $(\mathrm{C} 2)$ to $(\mathrm{C} 4)$ can be applied throughout regions $3 \mathrm{c}$ and 4.

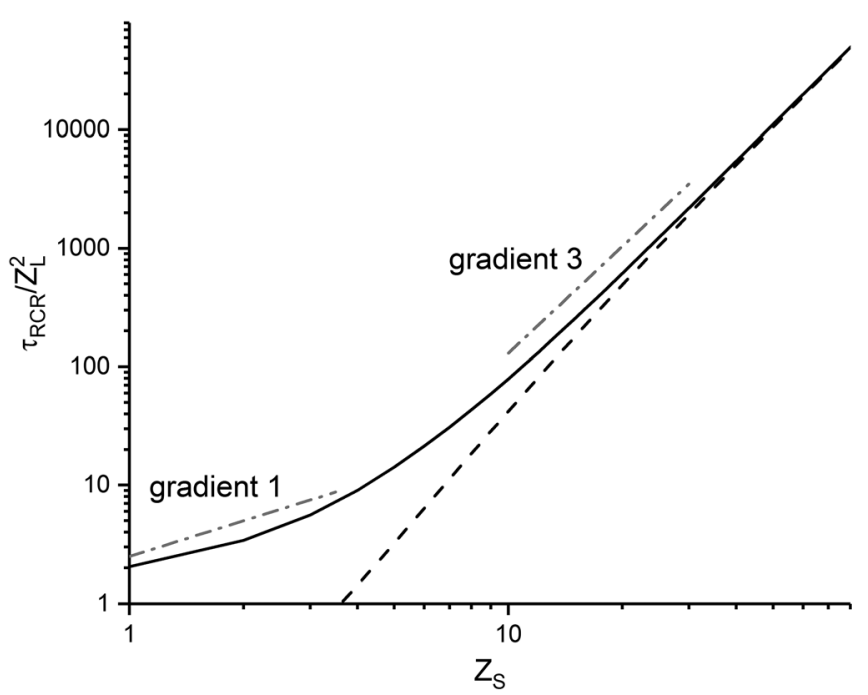

FIG. 9. $\tau_{\mathrm{RCR}} / Z_{L}^{2}$ versus $Z_{S}$ as evaluated using Eq. (C1) (solid line) and the leading order expression for slow $\mathrm{CR}, \tau_{\mathrm{RCR}}=Z_{L}^{2} \beta \tau_{\mathrm{dS}}$ (dashed line). Dashed dot lines have gradients of 1 and 3 in the logarithmic plot and are intended as guides to the eye. 
[1] de Gennes, P. G., "Reptation of a polymer chain in presence of fixed obstacles," J. Chem. Phys. 55, 572-579 (1971).

[2] Doi, M., and S. F. Edwards, The Theory of Polymer Dynamics (Clarendon, Oxford, 1986).

[3] Likhtman, A. E., "Single-chain slip-link model of entangled polymers: Simultaneous description of neutron spin-echo, rheology, and diffusion," Macromolecules 38, 6128-6139 (2005).

[4] Schieber, J. D., J. Neergaard, and, and S. Gupta, "A full-chain, temporary network model with sliplinks, chain-length fluctuations, chain connectivity and chain stretching," J. Rheol. 47, 213-233 (2003).

[5] Masubuchi, Y., J.-I. Takimoto, K. Koyama, G. Ianniruberto, G. Marrucci, and F. Greco, "Brownian simulations of a network of reptating primitive chains," J. Chem. Phys. 115, 4387-4394 (2001).

[6] Uneyama, T., and Y. Masubuchi, "Multi-chain slip-spring model for entangled polymer dynamics,” J. Chem. Phys. 137, 154902 (2012).

[7] Ramirez-Hernandez, A., B. L. Peters, M. Andreev, J. D. Schieber, and J. J. de Pablo, "A multichain polymer slip-spring model with fluctuating number of entanglements for linear and nonlinear rheology," J. Chem. Phys. 143, 243147 (2015).

[8] Dealy, J. M., D. J. Read, and R. G. Larson, Structure and Rheology of Molten Polymers: From Structure to Flow Behavior and Back Again (Carl Hanser Verlag GmbH \& Co. KG, Munchen, 2018).

[9] Likhtman, A. E., and T. C. B. McLeish, "Quantitative theory for linear dynamics of linear entangled polymers," Macromolecules 35, 6332-6343 (2002).

[10] des Cloizeaux, J., "Double reptation vs. simple reptation in polymer melts," Europhys. Lett. 5, 437-442 (1988).

[11] Read, D. J., K. Jagannathan, S. K. Sukumaran, and D. Auhl, "A fullchain constitutive model for bidisperse blends of linear polymers," J. Rheol. 56, 823-873 (2012).

[12] van Ruymbeke, E., V. Shchetnikava, Y. Matsumiya, and, and H. Watanabe, "Dynamic dilution effect in binary blends of linear polymers with well-separated molecular weights," Macromolecules 47, 7653-7665 (2014).

[13] Sawada, T., X. Qiao, and H. Watanabe, "Viscoelastic relaxation of linear polyisoprenes: Examination of constraint release mechanism," Nihon Reorojo Gakkaishi 35, 11-20 (2007).

[14] Park, S. J., and R. G. Larson, "Tube dilation and reptation in binary blends of monodisperse linear polymers," Macromolecules 37, 597-604 (2004).

[15] Park, S. J., and R. G. Larson, "Long-chain dynamics in binary blends of monodisperse linear polymers," J. Rheol. 50, 21-39 (2006).

[16] Watanabe, H., T. Sakamoto, and T. Kotaka, "Viscoelastic properties of binary blends of narrow molecular weight distribution polystyrenes 2," Macromolecules 18, 1008-1015 (1985).

[17] Watanabe, H., and T. Kotaka, "Viscoelastic properties and relaxation mechanisms of binary blends of narrow molecular weight distribution polystyrenes," Macromolecules 17, 2316-2325 (1985).

[18] Viovy, J. L., M. Rubinstein, and R. H. Colby, "Constraint release in polymer melts: Tube reorganization versus tube dilation," Macromolecules 24, 3587-3596 (1991).

[19] Struglinski, M. J., and W. W. Graessley, "Effects of polydispersity on the linear viscoelastic properties of entangled polymers. 1. Experimental observations for binary mixtures of linear polybutadiene," Macromolecules 18, 2630-2643 (1985).
[20] Daoud, M., and, and P. G. de Gennes, "Some remarks on the dynamics of polymer melts,” J. Polym. Sci. Polym. Phys. Ed. 17, 1971-1981 (1979).

[21] Shivokhin, M. E., D. J. Read, D. Kouloumasis, R. Kocen, F. Zhuge, C. Bailly, N. Hadjichristidis, and, and A. E. Likhtman, "Understanding effect of constraint release environment on end-to-end vector relaxation of linear polymer chains," Macromolecules 50, 4501-4523 (2017).

[22] Shahid, T., Q. Huang, F. Oosterlinck, C. Clasen, and, and E. van Ruymbeke, "Dynamic dilution exponent in monodisperse entangled polymer solutions," Soft Matter 13, 269-282 (2017).

[23] Shivokhin, M. E., E. van Ruymbeke, C. Bailly, D. Kouloumasis, N. Hadjichristidis, and A. E. Likhtman, "Understanding constraint release in star/linear polymer blends," Macromolecules 47, 2451-2463 (2014).

[24] Wang, Z., A. E. Likhtman, and R. G. Larson, "Segmental dynamics in entangled linear polymer melts," Macromolecules 45, 3557-3570 (2012).

[25] Ramirez, J., S. K. Sukumaran, B. Vorselaars, and, and A. E. Likhtman, "Efficient on the fly calculation of time correlation functions in computer simulations," J. Chem. Phys. 133, 154103 (2010).

[26] Matsumiya, Y., H. Watanabe, and K. Osaki, "Comparison of dielectric and viscoelastic relaxation functions of cis-polyisoprenes: Test of tube dilation molecular picture," Macromolecules 33, 499-506 (2000).

[27] Watanabe, H., Y. Matsumiya, and T. Inoue, "Dielectric and viscoelastic relaxation of highly entangled star polyisoprene: Quantitative test of tube dilation model," Macromolecules 35, 2339-2357 (2002).

[28] Auhl, D., P. Chambon, T. C. B. McLeish, and D. J. Read, "Elongational flow of blends of long and short polymers: Effective stretch relaxation time," Phys. Rev. Lett. 103, 136001 (2009).

[29] McLeish, T. C. B., "Tube theory of entangled polymer dynamics," Adv. Phys. 51, 1379-1527 (2002).

[30] McLeish, T. C. B., in Soft and Fragile Matter, edited by M. E. Cates and M. R. Evans (SUSSP Publications, Edinburgh / Institute of Physics Publishing, Bristol, U.K., 2000).

[31] Watanabe, H., S. Ishida, Y. Matsumiya, and T. Inoue, "Dielectric and viscoelastic behavior of entangled blends of polyisoprenes having widely separated molecular weights: Test of tube dilation picture," Macromolecules 37, 1937-1951 (2004).

[32] Watanabe, H., S. Ishida, Y. Matsumiya, and T. Inoue, "Test of full and partial tube dilation pictures in entangled blends of linear polyisoprenes," Macromolecules 37, 6619-6631 (2004).

[33] Lee, J. H., L. J. Fetters, L. A. Archer, and A. F. Halasa, "Dynamics of binary polymer blends," Macromolecules 38, 3917-3932 (2005).

[34] Rubinstein, M., and R. H. Colby, "Self-consistent theory of polydisperse entangled polymers: Linear viscoelasticity of binary blends," J. Chem. Phys. 89, 5291-5306 (1988).

[35] Wang, S., S. Wang, A. Halasa, and W.-L. Hsu, "Relaxation dynamics in mixtures of long and short chains: Tube dilation and impeded curvilinear diffusion," Macromolecules 36, 5355-5371 (2003).

[36] Montfort, J. P., G. Martin, J. Arman, and Ph. Monge, "Viscoelastic properties of high molecular weight polymers in the molten state II. Influence of the molecular weight distribution on linear viscoelastic properties," Rheol. Acta 18, 623-628 (1979).

[37] Ebrahimi, T., H. Taghipour, D. Grieß1, P. Mehrkhodavandi, S. G. Hatzikiriakos, and E. van Ruymbeke, "Binary blends of entangled star and linear poly(hydroxybutyrate): Effect of constraint release and dynamic tube dilation," Macromolecules 50, 2535-2546 (2017). 